\title{
Communication Efficient Decentralized Learning Over Bipartite Graphs
}

\author{
Chaouki Ben Issaid ${ }^{\circledR}$, Member, IEEE, Anis Elgabli ${ }^{\circledR}$, Member, IEEE, Jihong Park ${ }^{\circledR}$, Member, IEEE, \\ Mehdi Bennis ${ }^{\circledR}$, Fellow, IEEE, and Mérouane Debbah
}

\begin{abstract}
In this paper, we propose a communicationefficiently decentralized machine learning framework that solves a consensus optimization problem defined over a network of inter-connected workers. The proposed algorithm, Censored and Quantized Generalized GADMM (CQ-GGADMM), leverages the worker grouping and decentralized learning ideas of Group Alternating Direction Method of Multipliers (GADMM), and pushes the frontier in communication efficiency by extending its applicability to generalized network topologies, while incorporating link censoring for negligible updates after quantization. We theoretically prove that CQ-GGADMM achieves the linear convergence rate when the local objective functions are strongly convex under some mild assumptions. Numerical simulations corroborate that CQ-GGADMM exhibits higher communication efficiency in terms of the number of communication rounds and transmit energy consumption without compromising the accuracy and convergence speed, compared to the censored decentralized ADMM, and the worker grouping method of GADMM.
\end{abstract}

Index Terms-Alternating direction method of multipliers, communication efficiency, decentralized machine learning, stochastic quantization.

\section{INTRODUCTION}

$\mathbf{M}$ ACHINE learning is central to emerging missioncritical applications such as autonomous driving, remote surgery, and the fifth-generation $(5 \mathrm{G})$ communication systems and beyond [1]-[3]. These applications commonly require extremely low latency and high reliability while accurately reacting to local environmental dynamics [4]. To this end, training their machine learning models needs the sheer amount of fresh training data samples that are generated

Manuscript received January 7, 2021; revised June 2, 2021 and September 23, 2021; accepted October 31, 2021. Date of publication November 17, 2021; date of current version June 10, 2022. This work was supported in part by the Academy of Finland 6G Flagship under Grant 318927 and the Project SMARTER, in part by the Project EU-ICT IntellIoT under Grant 957218 and the Project EUCHISTERA LearningEdge, and in part by the CONNECT, Infotech-NOOR, and NEGEIN. The associate editor coordinating the review of this article and approving it for publication was $\mathrm{T}$. Q. S. Quek. (Corresponding author: Chaouki Ben Issaid.)

Chaouki Ben Issaid, Anis Elgabli, and Mehdi Bennis are with the Centre of Wireless Communications, University of Oulu, 90014 Oulu, Finland (e-mail: chaouki.benissaid@oulu.fi; anis.elgabli@oulu.fi; mehdi.bennis@oulu.fi).

Jihong Park is with the School of Information Technology, Deakin University, Geelong, VIC 3220, Australia (e-mail: jihong.park@ deakin.edu.au).

Mérouane Debbah is with the Technology Innovation Institute, Masdar City, Abu Dhabi, United Arab Emirates, and also with the CentraleSupélec, University of Paris-Saclay, 91192 Gif-sur-Yvette, France (e-mail: merouane.debbah@tii.ae).

Color versions of one or more figures in this article are available at https://doi.org/10.1109/TWC.2021.3126859.

Digital Object Identifier 10.1109/TWC.2021.3126859 by and dispersed across edge devices (e.g., phones, cars, access points, etc.), hereafter referred to as workers. Collecting these raw data may not only violate the data privacy, but also incur significant communication overhead under limited bandwidth. This calls for developing communication-efficient and privacy-preserving distributed learning frameworks [5], [6]. Federated learning is one representative method that ensures learning through periodically exchanging model parameters across workers rather than sending private data samples [7]. Nevertheless, federated learning postulates a parameter server collecting and distributing model parameters, which is not always accessible from faraway workers and is vulnerable to a single point of failure [8].

In this respect, fully decentralized learning frameworks are promising solutions in which workers directly communicate with each other without any central coordination [9]-[12]. However, due to the lack of the parameter server, the convergence speed is often too slow, particularly when some workers encounter extremely sparse connectivity. Indeed, existing decentralized learning frameworks such as distributed gradient descent (DGD) and multi-step primaldual (MSPD) frameworks can only achieve sub-linear convergence rates [9], [10]. Spurred by this motivation, the Group ADMM (GADMM) framework exploits the Alternating Direction Method of Multipliers algorithm to accelerate the convergence speed, and thereby achieves a linear convergence rate under a fully decentralized architecture with sparse connectivity [11]. Meanwhile, a quantization based GADMM (Q-GADMM) additionally utilizes stochastic quantization, and reduces communication payload sizes without compromising the convergence rate of GADMM [12]. Notwithstanding, both GADMM and Q-GADMM are limited to a ring network topology where each worker is connected only with at most two neighboring workers as shown in Fig. 1(a), questioning its scalability.

To fill this void, by generalizing and extending GADMM and Q-GADMM, in this article we propose a novel decentralized learning framework, coined Censored and Quantized Generalized Group ADMM (CQ-GGADMM) as illustrated in see Fig. 1(c). On the one hand, following GADMM, the workers in CQ-GGADMM are divided into head and tail groups in which the workers in the same group update their models in parallel, whereas the workers in different groups update their models in an alternating way. On the other hand, following Q-GADMM, CQ-GGADMM reduces 


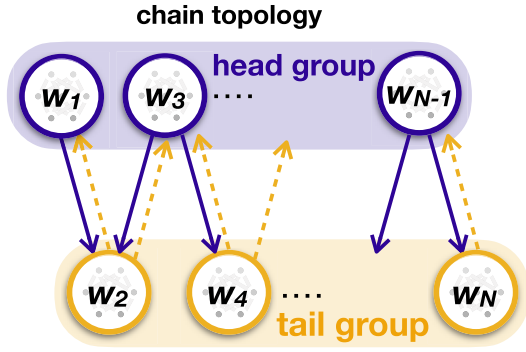

(a) GADMM

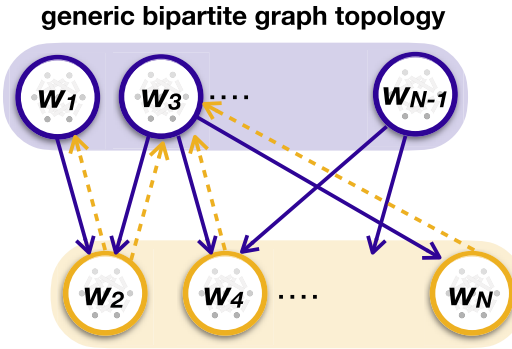

(b) GGADMM

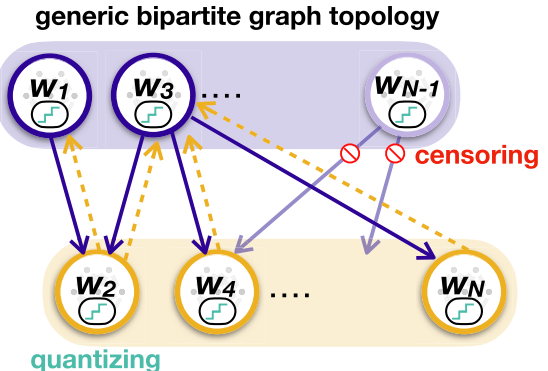

(c) CQ-GGADMM

Fig. 1. A schematic illustration of (a) group ADMM (GADMM), (b) generalized GADMM (GGADMM) and (c) censored and quantized GGADMM $(C Q-G G A D M M)$.

the communication payload size per each link by applying a heterogeneous stochastic quantization scheme that decreases the number of bits to represent each model parameter [12]. Furthermore, to reduce the number of communication links per round, CQ-GGADMM introduces a link censoring technique that allows to exchange model parameters only when the updated quantized model is sufficiently changed from the previous quantized model, i.e., skipping small quantized model updates [13]. While leveraging the aforementioned three principles, CQ-GADMM is built upon a generalized version of GADMM (GGADMM), wherein each worker can connect with an arbitrary number of neighbors under a generic bipartite graph topology as shown in Fig. 1(b). This is in contrast to the original GADMM that is operational only under a ring topology [11] as shown in Fig. 1(a).

\section{A. Related Works}

Towards improving the communication efficiency of distributed learning, prior works have studied various techniques under centralized and decentralized network architectures.

a) Fast Convergence: The total communication cost until completing a distributed learning operation can be reduced by accelerating the convergence speed. To this end, departing from the conventional first-order methods such as distributed gradient descent [14], primal-dual methods are applied under centralized [13], [15], [16] and decentralized architectures [11]. Furthermore, momentum acceleration is utilized under centralized [17], [18] and decentralized settings [19].

b) Link Sparsification: In large-scale distributed learning, a large portion of total communication links is often redundant [20]. In this respect, for each communication round, sparsifying the number of communication links can reduce the communication cost without compromising the accuracy. To this end, link censoring for negligible model updates is applied under centralized [21] and decentralized network topologies [11], [22].

c) Payload Size Reduction: To reduce the communication payload size per link, model updates are quantized under centralized [23]-[25] and decentralized network topologies [12], [19], [26]. Alternatively, the entries of model updates can be partially dropped as shown under centralized [27] and decentralized architectures [28]. Furthermore, under centralized settings, model parameters can be compressed at the parameter server via knowledge distillation (KD) or while training and running KD simultaneously, i.e., federated distillation [29], [30].

Although the aforementioned principles have been separately studied in [11]-[13], integrating them for maximizing the communication efficiency while guaranteeing fast convergence remains a non-trivial problem. Compared to [11], [12], we extend the topology of the network from a chain topology where each worker is allowed to communicate with at most two neighbours to a bipartite topology where no constraint is imposed on the number of neighbours. Furthermore, we consider incorporating temporal sparsity by leveraging the censoring idea introduced in [13] to make the algorithm more communication efficient. Finally, unlike the works [11], [12], detailed proof of the derivation of the convergence rate is included. Compared to [13], quantization is introduced to significantly increase the communication savings while guaranteeing the linear convergence of the proposed algorithm. By doing so, CQ-GGADMM achieves significant savings in terms of the total number of transmitted bits (and consequently in terms of the total energy) compared to [13]. Incorporating both censoring and quantization steps incur model update errors that may propagate over communication rounds due to the lack of central entity. To resolve this problem, we carefully determine the non-increasing target censoring threshold and quantization step size, such that the model updates are more finely tuned as time elapses until convergence. We thereby prove the linear convergence rate of CQ-GGADMM, and show its effectiveness by simulations, in terms of convergence speed, total communication cost, and transmission energy consumption.

d) Contributions: The major contributions of this work are summarized as follows.

- We propose CQ-GGADMM, a primal-dual decentralized learning framework utilizing censoring, quantization, and GADMM for any bipartite and connected network topology graph (Algorithm 1 in Sec. III).

- We prove that CQ-GGADMM converges to the optimal solution for convex loss functions (Theorem 1 in Sec. IV).

- We identify the network topology conditions under which CQ-GGADMM achieves a linear convergence rate (Theorem 2 in Sec. IV) when the loss functions are strongly convex. 
- Numerical simulations have corroborated that in linear and logistic regression tasks using synthetic and real datasets, CQ-GGADMM achieves the same convergence speed at significantly lower communication rounds and several orders of magnitude less transmission energy, compared to C-GGADMM and Censored ADMM (C-ADMM) in [13].

The remainder of this paper is organized as follows. In section II, we describe the generalized version of GADMM (GGADMM) for a bipartite and connected graph, and formulate the decentralized learning problem. Then, we extend GGADMM to censored and quantized GGADMM (CQ-GGADMM) in Section III. In Section IV, we prove the convergence of CQ-GGADMM theoretically under some mild conditions. Finally, Section V validates the performance of CQ-GGADMM by simulations. The details of the proofs of our results are deferred to the appendices.

\section{PROBLEM Formulation}

We consider a connected network wherein a set $\mathcal{V}$ of $N$ workers aim to reach a consensus around a solution of a global optimization problem. The problem is solved using only local data and information available for each worker. Moreover, communication is constrained to only take place between neighboring workers. The optimization problem is given by

$$
\text { (P1) } \boldsymbol{\Theta}^{*}:=\arg \min _{\boldsymbol{\Theta}} \sum_{n=1}^{N} f_{n}(\boldsymbol{\Theta}) \text {, }
$$

where $\Theta \in \mathbb{R}^{d \times 1}$ is the global model parameter and $f_{n}$ : $\mathbb{R}^{d} \rightarrow \mathbb{R}$ is a local function composed of data stored at worker $n$. Problem (P1) appears in many applications of machine learning, especially when the dataset is very large and the training is carried out using different workers. The connections among workers are represented as an undirected communication graph $\mathcal{G}$ having the set $\mathcal{E} \subseteq \mathcal{V} \times \mathcal{V}$ of edges. The set of neighbors of worker $n$ is defined as $\mathcal{N}_{n}=$ $\{m \mid(n, m) \in \mathcal{E}\}$ whose cardinality is $\left|\mathcal{N}_{n}\right|=d_{n}$. We start by making the following key assumption.

Assumption 1: The communication graph $\mathcal{G}$ is bipartite and connected.

Under Assumption 1, following the worker grouping of GADMM [11], workers are divided into two groups: a head group $\mathcal{H}$, and a tail group $\mathcal{T}$. Note that unlike [11] where every worker needs to connect with at most two neighbors under a chain network topology, each worker in CQ-GGADMM can connect with an arbitrary number of neighbors, as long as the network topology graph is bipartite and connected. Each head worker in $\mathcal{H}$ can only communicate with tail workers in $\mathcal{T}$, and vice versa. In this case, the edge set definition can be re-written as $\mathcal{E}=\{(n, m) \mid n \in \mathcal{H}, m \in \mathcal{T}\}$, and the problem (P1) is equivalent to the following problem

$$
\begin{array}{r}
\text { (P2) } \boldsymbol{\theta}^{*}:=\arg \min _{\left\{\boldsymbol{\theta}_{n}\right\}_{n=1}^{N}} \sum_{n=1}^{N} f_{n}\left(\boldsymbol{\theta}_{n}\right) \\
\text { s.t. } \boldsymbol{\theta}_{n}=\boldsymbol{\theta}_{m}, \quad \forall(n, m) \in \mathcal{E},
\end{array}
$$

where $\boldsymbol{\theta}_{n}$ is the local copy of the common optimization variable $\Theta$ at worker $n$. Note that, under the formulation (P2), the objective function becomes separable across the workers and as a consequence the problem can be solved in a distributed manner. In this case, the Lagrangian of the optimization problem (P2) can be written as

$$
\begin{array}{r}
\mathcal{L}_{\rho}(\boldsymbol{\theta}, \boldsymbol{\lambda})=\sum_{n=1}^{N} f_{n}\left(\boldsymbol{\theta}_{n}\right)+\sum_{(n, m) \in \mathcal{E}}\left\langle\boldsymbol{\lambda}_{n, m}, \boldsymbol{\theta}_{n}-\boldsymbol{\theta}_{m}\right\rangle \\
+\frac{\rho}{2} \sum_{(n, m) \in \mathcal{E}}\left\|\boldsymbol{\theta}_{n}-\boldsymbol{\theta}_{m}\right\|^{2},
\end{array}
$$

where $\rho>0$ is a constant penalty parameter and $\boldsymbol{\lambda}_{n, m}$ is the dual variable between neighboring workers $n$ and $m$, $\forall(n, m) \in \mathcal{E}$. At iteration $k+1$, the Generalized Group ADMM (GGADMM) algorithm runs as follows.

(1) Every head worker, $n \in \mathcal{H}$, updates its primal variable by solving

$$
\begin{aligned}
\boldsymbol{\theta}_{n}^{k+1}= & \underset{\boldsymbol{\theta}_{n}}{\arg \min } f_{n}\left(\boldsymbol{\theta}_{n}\right)+\sum_{m \in \mathcal{N}_{n}}\left\langle\boldsymbol{\lambda}_{n, m}^{k}, \boldsymbol{\theta}_{n}-\boldsymbol{\theta}_{m}^{k}\right\rangle \\
& +\frac{\rho}{2} \sum_{m \in \mathcal{N}_{n}}\left\|\boldsymbol{\theta}_{n}-\boldsymbol{\theta}_{m}^{k}\right\|^{2},
\end{aligned}
$$

and sends its updated model to its neighbors.

(2) The primal variables of tail workers, $m \in \mathcal{T}$, are then updated as

$$
\begin{aligned}
\boldsymbol{\theta}_{m}^{k+1}= & \underset{\boldsymbol{\theta}_{m}}{\arg \min } f_{m}\left(\boldsymbol{\theta}_{m}\right)+\sum_{n \in \mathcal{N}_{m}}\left\langle\boldsymbol{\lambda}_{n, m}^{k}, \boldsymbol{\theta}_{n}^{k+1}-\boldsymbol{\theta}_{m}\right\rangle \\
& +\frac{\rho}{2} \sum_{n \in \mathcal{N}_{m}}\left\|\boldsymbol{\theta}_{n}^{k+1}-\boldsymbol{\theta}_{m}\right\|^{2} .
\end{aligned}
$$

(3) The dual variables are updated locally for every worker, after receiving the model updates from its neighbors, in the following way

$$
\boldsymbol{\lambda}_{n, m}^{k+1}=\boldsymbol{\lambda}_{n, m}^{k}+\rho\left(\boldsymbol{\theta}_{n}^{k+1}-\boldsymbol{\theta}_{m}^{k+1}\right), \quad \forall(n, m) \in \mathcal{E} .
$$

Note that GGADMM is a generalized version of GADMM algorithm proposed in [11] since it considers an arbitrary topology. Introducing $\boldsymbol{\alpha}_{n}=\sum_{m \in \mathcal{N}_{n}} \boldsymbol{\lambda}_{n, m}, \forall n \in \mathcal{V}$, we can write

(1) The update of the models of head workers is done in parallel by solving

$$
\begin{aligned}
\boldsymbol{\theta}_{n}^{k+1}= & \underset{\boldsymbol{\theta}_{n}}{\arg \min } f_{n}\left(\boldsymbol{\theta}_{n}\right)+\left\langle\boldsymbol{\theta}_{n}, \boldsymbol{\alpha}_{n}^{k}-\rho \sum_{m \in \mathcal{N}_{n}} \boldsymbol{\theta}_{m}^{k}\right\rangle \\
& +\frac{\rho}{2} d_{n}\left\|\boldsymbol{\theta}_{n}\right\|^{2} .
\end{aligned}
$$

(2) The models of tail workers are updated in parallel using

$$
\begin{aligned}
\boldsymbol{\theta}_{m}^{k+1}= & \underset{\boldsymbol{\theta}_{m}}{\arg \min } f_{m}\left(\boldsymbol{\theta}_{m}\right)+\left\langle\boldsymbol{\theta}_{m}, \boldsymbol{\alpha}_{m}^{k}-\rho \sum_{n \in \mathcal{N}_{m}} \boldsymbol{\theta}_{n}^{k+1}\right\rangle \\
& +\frac{\rho}{2} d_{m}\left\|\boldsymbol{\theta}_{m}\right\|^{2} .
\end{aligned}
$$

(3) Instead of updating $\boldsymbol{\lambda}_{n, m}$, each worker will update locally the new auxiliary variable $\boldsymbol{\alpha}_{n}$ as

$$
\boldsymbol{\alpha}_{n}^{k+1}=\boldsymbol{\alpha}_{n}^{k}+\rho \sum_{m \in \mathcal{N}_{n}}\left(\boldsymbol{\theta}_{n}^{k+1}-\boldsymbol{\theta}_{m}^{k+1}\right), \quad \forall n \in \mathcal{V}
$$




\section{Censored Quantized Generalized GROUP ADMM}

To reduce the communication payload size, we follow a similar stochastic quantization scheme to the one described in [12]. Each worker $n$ quantizes the difference between its current model and its previously quantized model before transmission as $\boldsymbol{\theta}_{n}^{k}-\hat{\boldsymbol{Q}}_{n}^{k-1}=Q_{n}\left(\boldsymbol{\theta}_{n}^{k}, \hat{\boldsymbol{Q}}_{n}^{k-1}\right)$, where $\hat{\boldsymbol{Q}}_{n}^{k-1}$ is the quantized model at iteration $(k-1)$ and $Q_{n}(\cdot)$ is a stochastic quantization operator that depends on the quantization probability $p_{n, i}^{k}$ for each model vector's dimension $i \in\{1,2, \cdots, d\}$, and on $b_{n}^{k}$ bits used for representing each model vector dimension.

The $i^{\text {th }}$ dimensional element $\left[\hat{\boldsymbol{Q}}_{n}^{k-1}\right]_{i}$ of the previously quantized model vector is centred at the quantization range $2 R_{n}^{k}$ that is equally divided into $\left(2^{b_{n}^{k}}-1\right)$ quantization levels, yielding the quantization step size $\Delta_{n}^{k}=2 R_{n}^{k} /\left(2^{b_{n}^{k}}-1\right)$. In this coordinate, the difference between the $i^{\text {th }}$ dimensional element $\left[\boldsymbol{\theta}_{n}^{k}\right]_{i}$ of the current model vector and $\left[\hat{\boldsymbol{Q}}_{n}^{k-1}\right]_{i}$ is

$$
\left[c_{n}\left(\boldsymbol{\theta}_{n}^{k}\right)\right]_{i}=\frac{1}{\Delta_{n}^{k}}\left(\left[\boldsymbol{\theta}_{n}^{k}\right]_{i}-\left[\hat{\boldsymbol{Q}}_{n}^{k-1}\right]_{i}+R_{n}^{k}\right),
$$

where $R_{n}^{k}$ ensures the non-negativity of the quantized value. Then, $\left[c_{n}\left(\boldsymbol{\theta}_{n}^{k}\right)\right]_{i}$ is mapped to

$$
\left[q_{n}\left(\boldsymbol{\theta}_{n}^{k}\right)\right]_{i}=\left\{\left[\begin{array}{ll}
{\left[c_{n}\left(\boldsymbol{\theta}_{n}^{k}\right)\right]_{i}} \\
{\left[c_{n}\left(\boldsymbol{\theta}_{n}^{k}\right)\right]_{i}}
\end{array}\right] \begin{array}{l}
\text { with probability } p_{n, i}^{k} \\
\text { with probability } 1-p_{n, i}^{k}
\end{array}\right.
$$

where $[\cdot]$ and $\lfloor\cdot \mid$ are the ceiling and floor functions, respectively. Next, the probability $p_{n, i}^{k}$ in (11) is selected such that the expected quantization error $\mathbb{E}\left[\boldsymbol{e}_{n, i}^{k}\right]$ is zero

$$
p_{n, i}^{k}=\left(\left[c_{n}\left(\boldsymbol{\theta}_{n}^{k}\right)\right]_{i}-\left\lfloor\left[c_{n}\left(\boldsymbol{\theta}_{n}^{k}\right)\right]_{i}\right\rfloor\right) .
$$

The choice of $p_{n, i}^{k}$ in (12) ensures that the quantization in (11) is unbiased and the quantization error variance $\mathbb{E}\left[\left(\boldsymbol{e}_{n, i}^{k}\right)^{2}\right]$ is less than $\left(\Delta_{n}^{k}\right)^{2}$. This implies that $\mathbb{E}\left[\left\|\boldsymbol{e}_{n}^{k}\right\|^{2}\right] \leq$ $d\left(\Delta_{n}^{k}\right)^{2}$.

The convergence of CQ-GGADMM requires non-increasing quantization step sizes over iterations, i.e., $\Delta_{n}^{k} \leq \omega \Delta_{n}^{k-1}$ for all $k$ where $\omega \in(0,1)$. To satisfy this condition, the parameter $b_{n}^{k}$ is chosen as

$$
b_{n}^{k} \geq\left\lceil\log _{2}\left(1+\left(2^{b_{n}^{k-1}}-1\right) R_{n}^{k} /\left(\omega R_{n}^{k-1}\right)\right)\right\rceil .
$$

Under this condition, we get that $\Delta_{n}^{k} \leq \omega^{k} \Delta_{n}^{0}$. With the aforementioned stochastic quantization procedure, $b_{n}^{k}$, $R_{n}^{k}$, and $q_{n}\left(\boldsymbol{\theta}_{n}^{k}\right)$ suffice to represent $\hat{\boldsymbol{Q}}_{n}^{k}$, where $q_{n}\left(\boldsymbol{\theta}_{n}^{k}\right)=$ $\left(\left[q_{n}\left(\boldsymbol{\theta}_{n}^{k}\right)\right]_{1}, \ldots,\left[q_{n}\left(\boldsymbol{\theta}_{n}^{k}\right)\right]_{d}\right)^{\boldsymbol{\top}}$ which are transmitted to neighbors. After receiving these values, $\hat{\boldsymbol{Q}}_{n}^{k}$ can be reconstructed as [31], [32]

$$
\hat{\boldsymbol{Q}}_{n}^{k}=\hat{\boldsymbol{Q}}_{n}^{k-1}+\Delta_{n}^{k} q_{n}\left(\boldsymbol{\theta}_{n}^{k}\right)-R_{n}^{k} \mathbf{1} .
$$

Now, we introduce a censoring condition that reduces the communication overhead by reducing the number of workers communicating at a given iteration. Under this condition, the worker is allowed to transmit only when the difference between the current and previously transmitted value is sufficiently different. However, we apply the censoring not on the model itself but on its quantized value, i.e., if the worker is not censored, it transmits its quantized model to its neighbors. According to the communication-censoring strategy, we have that $\hat{\boldsymbol{\theta}}_{n}^{k+1}=\hat{\boldsymbol{Q}}_{n}^{k+1}$ provided that $\left\|\hat{\boldsymbol{\theta}}_{n}^{k}-\hat{\boldsymbol{Q}}_{n}^{k+1}\right\| \geq \tau_{0} \xi^{k+1}$ and $\hat{\boldsymbol{\theta}}_{n}^{k+1}=\hat{\boldsymbol{\theta}}_{n}^{k}$, otherwise. The CQ-GGADMM algorithm can be written in this case as

(1) Primal variables for head workers are found using

$$
\begin{aligned}
\boldsymbol{\theta}_{n}^{k+1}=\underset{\boldsymbol{\theta}_{n}}{\arg \min } f_{n}\left(\boldsymbol{\theta}_{n}\right)+\left\langle\boldsymbol{\theta}_{n}, \boldsymbol{\alpha}_{n}^{k}-\rho \sum_{m \in \mathcal{N}_{n}} \hat{\boldsymbol{\theta}}_{m}^{k}\right\rangle \\
+\frac{\rho}{2} d_{n}\left\|\boldsymbol{\theta}_{n}\right\|^{2} .
\end{aligned}
$$

(2) Primal variables update for tail workers is done as follow

$$
\begin{aligned}
\boldsymbol{\theta}_{m}^{k+1}=\underset{\boldsymbol{\theta}_{m}}{\arg \min } f_{m}\left(\boldsymbol{\theta}_{m}\right)+\left\langle\boldsymbol{\theta}_{m}, \boldsymbol{\alpha}_{m}^{k}-\rho \sum_{n \in \mathcal{N}_{m}} \hat{\boldsymbol{\theta}}_{n}^{k+1}\right\rangle \\
+\frac{\rho}{2} d_{m}\left\|\boldsymbol{\theta}_{m}\right\|^{2} .
\end{aligned}
$$

(3) Dual variable of each worker is updated locally

$$
\boldsymbol{\alpha}_{n}^{k+1}=\boldsymbol{\alpha}_{n}^{k}+\rho \sum_{m \in \mathcal{N}_{n}}\left(\hat{\boldsymbol{\theta}}_{n}^{k+1}-\hat{\boldsymbol{\theta}}_{m}^{k+1}\right), \quad \forall n \in \mathcal{V} .
$$

Given $p_{n, i}^{k}$ in (12) and $b_{n}^{k}$ in (13), the convergence of CQ-GGADMM is provided in Section IV. Note that when the full arithmetic precision uses 32 bits, every transmission payload size of CQ-GGADMM is $b_{n}^{k} d+\left(b_{R}+b_{b}\right)$ bits, where $b_{R} \leq 32$ and $b_{b} \leq 32$ are the required bits to represent $R_{n}^{k}$ and $b_{n}^{k}$, respectively. Compared to GGADMM, whose payload size is $32 d$ bits, CQ-GGADMM can achieve a huge reduction in communication overhead, particularly for large models, i.e., large $d$.

\section{Convergence Analysis}

Before stating the main results of the paper, we further make the following assumptions.

Assumption 2: There exists an optimal solution set to (P1) which has at least one finite element.

Assumption 3: The local cost functions $f_{n}$ are convex.

Assumption 4: The local cost functions $f_{n}$ are strongly convex with parameter $\mu_{n}>0$, i.e.,

$$
\left\|\nabla f_{n}(\boldsymbol{x})-\nabla f_{n}(\boldsymbol{y})\right\| \geq \mu_{n}\|\boldsymbol{x}-\boldsymbol{y}\|, \quad \forall \boldsymbol{x}, \boldsymbol{y} \in \mathbb{R}^{d} .
$$

Assumption 5: The local cost functions $f_{n}$ have $L_{n^{-}}$ Lipschitz continuous gradient $\left(L_{n}>0\right)$

$$
\left\|\nabla f_{n}(\boldsymbol{x})-\nabla f_{n}(\boldsymbol{y})\right\| \leq L_{n}\|\boldsymbol{x}-\boldsymbol{y}\|, \quad \forall \boldsymbol{x}, \boldsymbol{y} \in \mathbb{R}^{d} .
$$

Assumptions 1-5 are key assumptions that are often used in the context of distributed optimization [13], [15], [21]. While only assumptions 1-3 are needed to prove the convergence of CQ-GGADMM, assumptions 4 and 5 are further required to show the linear convergence rate. Note that Assumption 2 ensures that the problem (P2) has at least one optimal solution, denoted by $\boldsymbol{\theta}^{\star}$. Under Assumption 4, the function $f$ is strongly convex with parameter $\mu=\min _{1 \leq n \leq N} \mu_{n}$, and from 


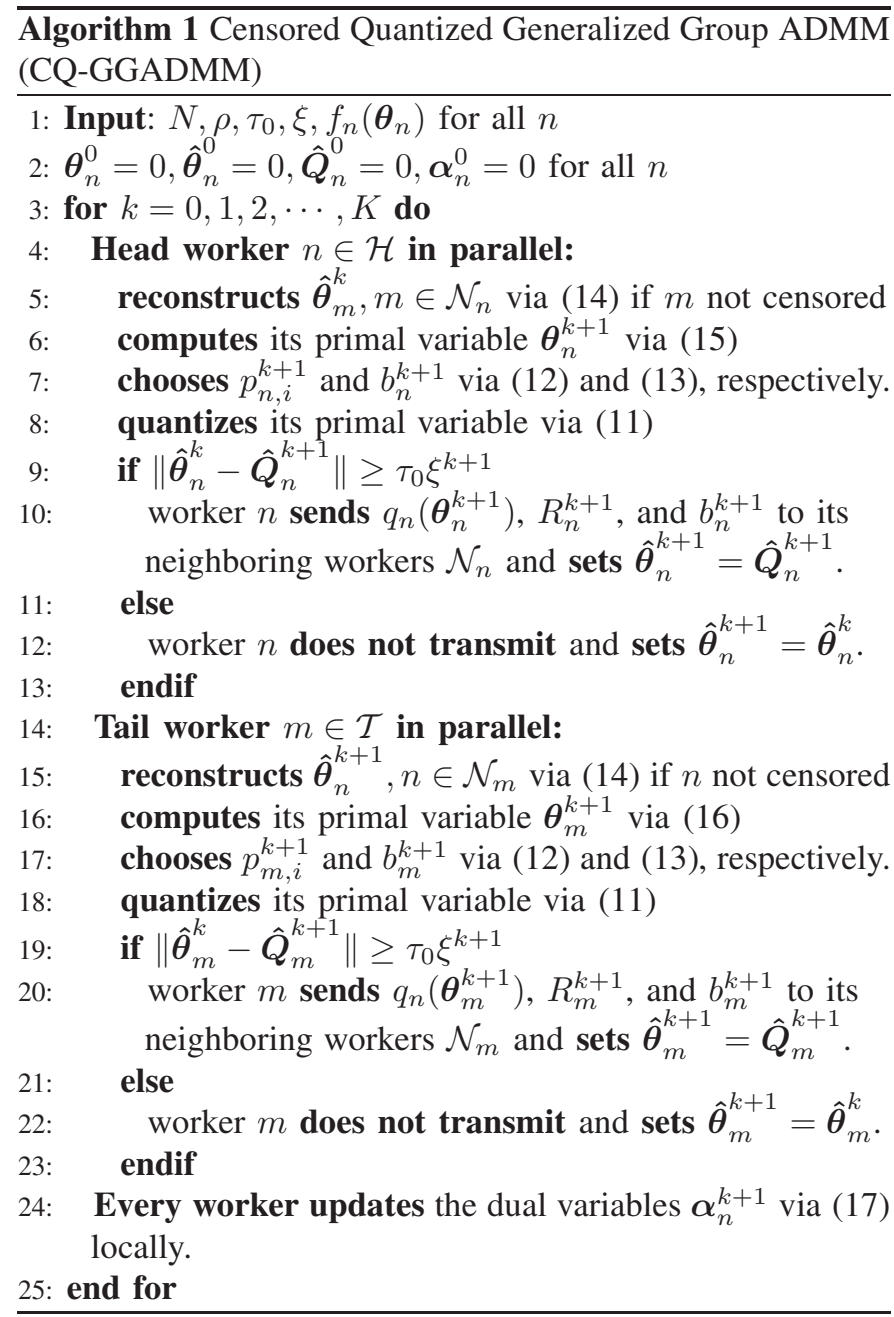

Assumption 5, we can see that $f$ has $L$-Lipschitz continuous gradient with $L=\max _{1 \leq n \leq N} L_{n}$.

To proceed with the analysis, we start by writing the optimality conditions as

$\boldsymbol{\theta}_{n}^{\star}=\boldsymbol{\theta}_{m}^{\star}, \quad \forall(n, m) \in \mathcal{E}$ and $\nabla f_{n}\left(\boldsymbol{\theta}_{n}^{\star}\right)+\boldsymbol{\alpha}_{n}^{\star}=\mathbf{0}, \quad \forall n \in \mathcal{V}$,

where $\boldsymbol{\theta}_{n}^{\star}$ and $\boldsymbol{\alpha}_{n}^{\star}$ are the optimal values of the primal and dual variables, respectively. We define the primal residual as

$$
\boldsymbol{r}_{n, m}^{k+1}=\boldsymbol{\theta}_{n}^{k+1}-\boldsymbol{\theta}_{m}^{k+1}, \quad \forall(n, m) \in \mathcal{E},
$$

and the dual residual as

$$
\boldsymbol{s}_{n}^{k+1}=\rho \sum_{m \in \mathcal{N}_{n}}\left(\hat{\boldsymbol{\theta}}_{m}^{k+1}-\hat{\boldsymbol{\theta}}_{m}^{k}\right), \quad \forall n \in \mathcal{H} .
$$

Finally, the total error is defined as

$$
\boldsymbol{\epsilon}_{n}^{k+1}=\boldsymbol{\theta}_{n}^{k+1}-\hat{\boldsymbol{\theta}}_{n}^{k+1}, \quad \forall n=1, \ldots, N
$$

The total error can be decomposed as the sum of two errors

(i) a random error coming from the quantization process $\boldsymbol{e}_{n}^{k+1}=\boldsymbol{\theta}_{n}^{k+1}-\hat{\boldsymbol{Q}}_{n}^{k+1}$

(ii) a deterministic one due to the censoring strategy $\ell_{n}^{k+1}=$ $\hat{\boldsymbol{Q}}_{n}^{k+1}-\hat{\boldsymbol{\theta}}_{n}^{k+1}$.
According to the communication-censoring strategy, we have that $\hat{\boldsymbol{\theta}}_{n}^{k}=\hat{\boldsymbol{Q}}_{\eta}^{k}$ if $\left\|\hat{\boldsymbol{\theta}}_{n}^{k-1}-\hat{\boldsymbol{Q}}_{n}^{k}\right\| \geq \tau_{0} \xi^{k}$ and $\hat{\boldsymbol{\theta}}_{n}^{k}=\hat{\boldsymbol{\theta}}_{n}^{k-1}$ if $\left\|\hat{\boldsymbol{\theta}}_{n}^{k-1}-\hat{\boldsymbol{Q}}_{n}^{k}\right\|<\tau_{0} \xi^{k}$. In both cases, we have $\left\|\boldsymbol{\ell}_{n}^{k}\right\|=$ $\left\|\hat{\boldsymbol{Q}}_{n}^{k}-\hat{\boldsymbol{\theta}}_{n}^{k}\right\|<\tau_{0} \xi^{k}$. Since the sequence $\left\{\tau_{0} \xi^{k}\right\}$ is a decreasing non-negative sequence, then we have that $\left\|\ell_{n}^{k}\right\| \leq \tau_{0} \xi^{k}$ and $\left\|\ell_{n}^{k+1}\right\| \leq \tau_{0} \xi^{k}, \forall n \in \mathcal{V}$. Since the second moment of the quantization error is bounded by $\mathbb{E}\left[\left\|e_{n}^{k}\right\|^{2}\right] \leq d\left(\Delta_{n}^{k}\right)^{2} \leq$ $d\left(\Delta^{0}\right)^{2} \omega^{2 k}$ where $\Delta^{0}=\max _{1<n<N} \Delta_{n}^{0}$, then, the total error can be upper bounded, using (30), by

$$
\begin{aligned}
\mathbb{E}\left[\left\|\boldsymbol{\epsilon}_{n}^{k}\right\|^{2}\right] & \leq 2\left(\left\|\boldsymbol{\ell}_{n}^{k}\right\|^{2}+\mathbb{E}\left[\left\|\boldsymbol{e}_{n}^{k}\right\|^{2}\right]\right) \\
& \leq 2\left(\tau_{0}^{2} \xi^{2 k}+d\left(\Delta^{0}\right)^{2} \omega^{2 k}\right) \leq 4 C_{0}^{2} \psi^{2 k}
\end{aligned}
$$

where $C_{0}=\max \left\{\tau_{0}, \sqrt{d}\left(\Delta^{0}\right)\right\}$, and $\psi=\max \{\xi, \omega\} \in(0,1)$.

To prove the convergence of the proposed algorithm, we start by stating and proving the first lemma where we derive upper and lower bounds on the expected value of the optimality gap.

Lemma 1: Under assumptions 1-3, we have the following bounds on the expected value of the optimality gap

$$
\begin{aligned}
& \text { (i) } \sum_{n=1}^{N} \mathbb{E}\left[f_{n}\left(\boldsymbol{\theta}^{k+1}\right)-f_{n}\left(\boldsymbol{\theta}^{\star}\right)\right] \\
& \leq-\sum_{(n, m) \in \mathcal{E}} \mathbb{E}\left[\left\langle\boldsymbol{\lambda}_{n, m}^{k+1}, \boldsymbol{r}_{n, m}^{k+1}\right\rangle\right]+\sum_{n \in \mathcal{H}} \mathbb{E}\left[\left\langle\boldsymbol{s}_{n}^{k+1}, \boldsymbol{\theta}_{n}^{\star}-\boldsymbol{\theta}_{n}^{k+1}\right\rangle\right] \\
& \quad+\rho \sum_{n=1}^{N} d_{n} \mathbb{E}\left[\left\langle\boldsymbol{\epsilon}_{n}^{k+1}, \boldsymbol{\theta}_{n}^{\star}-\boldsymbol{\theta}_{n}^{k+1}\right\rangle\right], \\
& \text { (ii) } \sum_{n=1}^{N} \mathbb{E}\left[f_{n}\left(\boldsymbol{\theta}^{k+1}\right)-f_{n}\left(\boldsymbol{\theta}^{\star}\right)\right] \geq-\sum_{(n, m) \in \mathcal{E}} \mathbb{E}\left[\left\langle\boldsymbol{\lambda}_{n, m}^{\star}, \boldsymbol{r}_{n, m}^{k+1}\right\rangle\right] .
\end{aligned}
$$

Proof: The details of the proof are deferred to Appendix VII-B.

Next, we present the first theorem that states the asymptotic convergence of the proposed algorithm where we prove the convergence to zero in the mean square sense of both the primal and dual residuals as well as the convergence to zero in the mean sense of the optimality gap.

Theorem 1: Suppose assumptions 1-3 hold, then the CQ-GGADMM iterates lead to

(i) the convergence of the primal residual to zero in the mean square sense as $k \rightarrow \infty$, i.e.,

$$
\lim _{k \rightarrow \infty} \mathbb{E}\left[\left\|\boldsymbol{r}_{n, m}^{k}\right\|^{2}\right]=0, \quad \forall(n, m) \in \mathcal{E},
$$

(ii) the convergence of the dual residual to zero in the mean square sense as $k \rightarrow \infty$, i.e.,

$$
\lim _{k \rightarrow \infty} \mathbb{E}\left[\left\|s_{n}^{k}\right\|^{2}\right]=0, \quad \forall n \in \mathcal{H},
$$

(iii) the convergence of the optimality gap to zero in the mean sense as $k \rightarrow \infty$, i.e.,

$$
\lim _{k \rightarrow \infty} \sum_{n=1}^{N} \mathbb{E}\left[f_{n}\left(\boldsymbol{\theta}_{n}^{k}\right)-f_{n}\left(\boldsymbol{\theta}_{n}^{\star}\right)\right]=0 .
$$

Proof: The proof can be found in Appendix VI-C. 
The linear convergence of the CQ-GGADMM algorithm is presented next.

Theorem 2: Suppose that assumptions 1, 2, 4 and 5 hold and the dual variable $\boldsymbol{\alpha}$ is initialized such that $\boldsymbol{\alpha}^{0}$ lies in the column space of the oriented incidence matrix $M_{-}$. For sufficiently small $\kappa$ and $\rho$, the sequence of iterates of $C Q-G G A D M M$ converges linearly with a rate $\left(1+\delta_{2}\right) / 2$ where $\delta_{2}=\max \left\{(1+\kappa)^{-1}, \psi^{2}\right\}$.

Proof: The proof is provided in Appendix VI-D where the conditions on $\kappa$ and $\rho$ are derived. In the proof, we require an extra initialization condition that $\boldsymbol{\alpha}^{0}$ lies in the column space of $\boldsymbol{M}_{-}$, by taking $\boldsymbol{\alpha}^{0}=\mathbf{0}$. Thus, we ensure that $\boldsymbol{\alpha}^{k}$ will always stay in the column space of $\boldsymbol{M}_{-}$and we can write $\boldsymbol{\alpha}^{k}=\boldsymbol{M}_{-} \boldsymbol{\beta}^{k}$. The convergence rate, derived in the proof, depends on the network topology through the values of $\sigma_{\max }(\boldsymbol{C}), \sigma_{\max }\left(\boldsymbol{M}_{-}\right)$and $\tilde{\sigma}_{\min }\left(\boldsymbol{M}_{-}\right)$, the properties of the local objective functions ( $\mu$ and $L$ ), the penalty parameter $\rho$ but also on the threshold parameter $\xi$ as well as the parameter $\omega$ used to construct the quantization step sizes.

\section{Numerical Results}

To validate our theoretical results, we numerically evaluate the performance of CQ-GGADMM compared with GGADMM, C-GGADMM, and C-ADMM [13]. Note that C-ADMM performs censoring on top of the Jacobian and decentralized version of the standard ADMM. For the tuning parameters, we choose the values leading to the best performance of all algorithms.

\section{A. Model and Datasets}

All simulations are conducted using synthetic and real datasets. For the synthetic data, we used the datasets that were generated in [21]. We consider two decentralized consensus optimization problems: $(i)$ linear regression, and (ii) logistic regression. The details about the datasets used in our experiments are summarized in Table I. For each dataset, the number of samples are uniformly distributed across the $N$ workers.

\section{B. Graph Generation}

Similarly to [33], we generate randomly a network consisting of $N$ workers with a connectivity ratio $p$. The ratio $p$ is defined as the actual number of edges divided by the number of edges for a fully connected graph, i.e., $N \times(N-1) / 2$. Such a random graph is created with $N p \times(N-1) / 2$ edges that are uniformly randomly chosen, while ensuring that the generated network is connected. Smaller values of $p$ leads to a sparser graph, while the generated graph becomes denser as $p$ approaches 1.

\section{Communication Energy}

We assume that the total system bandwidth $2 \mathrm{MHz}$ is equally divided across workers. Therefore, the available bandwidth to the $n$-th worker $\left(B_{n}\right)$ at every communication round when utilizing GGADMM is $(4 / N) \mathrm{MHz}$ since only half of the workers are transmitting at each communication round. On the other hand, the available bandwidth to each worker when using
C-ADMM is $(2 / N) \mathrm{MHz}$. The power spectral density $\left(N_{0}\right)$ is $10^{-6} \mathrm{~W} / \mathrm{Hz}$, and each upload/download transmission time $(\tau)$ is $1 \mathrm{~ms}$. We assume a free space model, and each worker needs to transmit at a power level that allows transmitting the model vector in one communication round (the rate is bottlenecked by the worst link). For example, using C-ADMM, each worker needs to find the transmission power that achieves the transmission rate $R=(32 d / 1 m s)$ bits/sec. Using Shannon capacity, the corresponding transmission power can be calculated as $P=\tau D^{2} N_{0} B_{n}\left(2^{R / B_{n}}-1\right)$, where $D$ is the distance to the farthest neighbor. Hence, the consumed energy will be $E=P \tau$.

\section{Linear Regression}

In this case, the local cost function at worker $n$ is explicitly given by $f_{n}(\boldsymbol{\theta})=\frac{1}{2}\left\|\boldsymbol{X}_{n} \boldsymbol{\theta}-\boldsymbol{y}_{n}\right\|^{2}$ where $\boldsymbol{X}_{n} \in \mathbb{R}^{s \times d}$ and $\boldsymbol{y}_{n} \in \mathbb{R}^{s \times 1}$ are private for each worker $n \in \mathcal{V}$ where $s$ represents the size of the data at each worker. Figs. 2-(a) and 3-(a) corroborate that both C-GGADMM and CQ-GGADMM achieve the same convergence speed as GGADMM and significantly outperform C-ADMM, thanks to the alternation update, censoring, and stochastic quantization. Note that though, C-ADMM allows workers to update their models in parallel, it requires significantly higher number of iterations. Figs. 2-(b) and 3-(b) show that C-GGADMM achieves $10^{-4}$ objective error with the minimum number of communication rounds outperforming all other algorithms. We also note that introducing quantization on top of censoring has increased the number of communication rounds. However, in terms of the total number of transmitted bits and consumed energy, CQ-GGADMM outperforms all algorithms.

\section{E. Logistic Regression}

In this section, we consider the binary logistic regression problem. We assume that worker $n$ owns a data matrix $\boldsymbol{X}_{n}=\left(\boldsymbol{x}_{n, 1}, \ldots, \boldsymbol{x}_{n, s}\right)^{T} \in \mathbb{R}^{s \times d}$ along with the corresponding labels $\boldsymbol{y}_{n}=\left(y_{n, 1}, \ldots, y_{n, s}\right) \in$ $\{-1,1\}^{s}$. The local cost function for worker $n$ is then given by $f_{n}(\boldsymbol{\theta})=\frac{1}{s} \sum_{j=1}^{s} \log \left(1+\exp \left(-y_{n, j} \boldsymbol{x}_{n, j}^{T} \boldsymbol{\theta}\right)\right)+$ $\frac{\mu_{0}}{2}\|\boldsymbol{\theta}\|^{2}$ where $\mu_{0}$ is the regularization parameter. As observed from Figs. 4-(a) and 5-(a), C-GADMM requires more iterations compared to GADMM to achieve the same loss which leads to either no saving in the number of communication rounds (see Fig. 4-(b)) or a small saving in the number of communication rounds (see Fig. 5-(b)). It also appears that the update of each individual worker when not quantizing is important at each iteration and censoring hurts the convergence speed. However, interestingly, when introducing stochastic quantization and performing censoring on top of the quantized models, we overcome this issue, and we show significant savings in the number of communication rounds and the communication overhead per iteration.

\section{F. Impact of the Network Graph Density}

To study how the network graph density (the node degree) affects the performance of the proposed approach, we conduct 

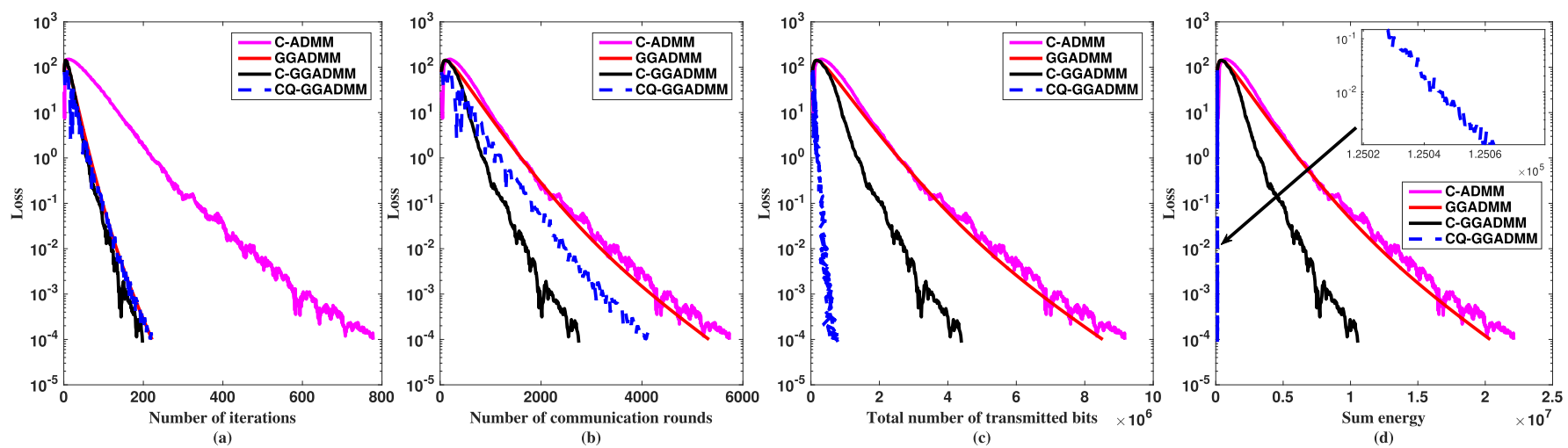

Fig. 2. Linear regression results on synthetic dataset showing the loss w.r.t.: (a) \# iterations; (b) \# communication rounds; (c) \# transmitted bits; (d) total energy.
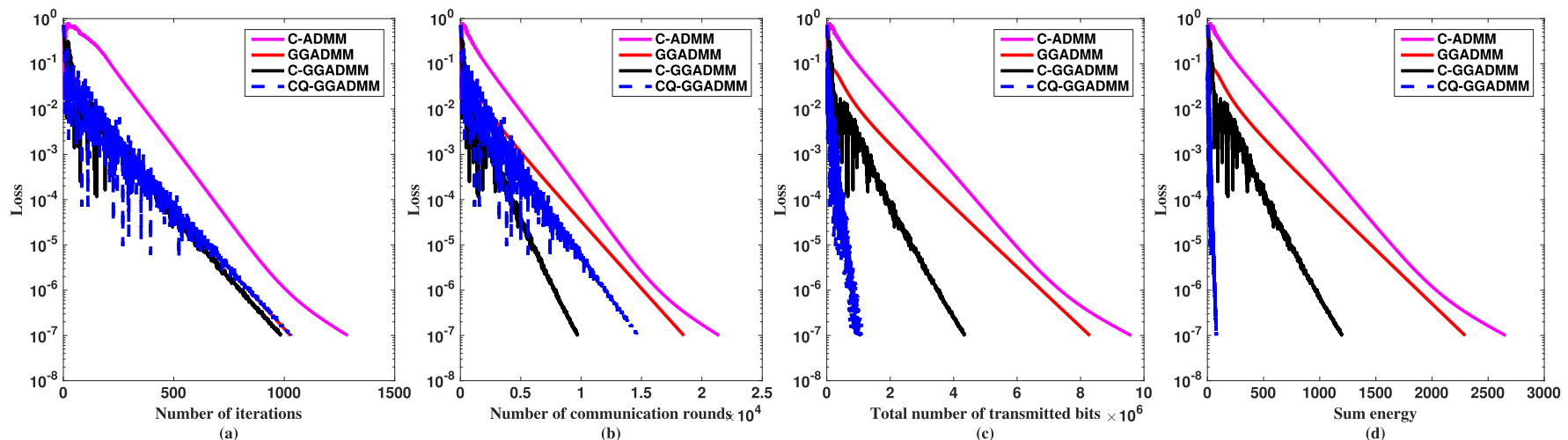

Fig. 3. Linear regression results on real dataset showing the loss w.r.t.: (a) \# iterations; (b) \# communication rounds; (c) \# transmitted bits; (d) total energy.

TABLE I

LIST OF DATASETS USED IN THE NUMERICAL EXPERIMENTS

\begin{tabular}{|l|l|l|c|c|}
\hline Dataset & Task & Data Type & Model Size $(d)$ & Number of Instances \\
\hline \hline synth-linear [21] & linear regression & synthetic & 50 & 1200 \\
\hline Body Fat [34] & linear regression & real & 14 & 252 \\
\hline synth-logistic [21] & logistic regression & synthetic & 50 & 1200 \\
\hline Derm [34] & logistic regression & real & 34 & 358 \\
\hline
\end{tabular}

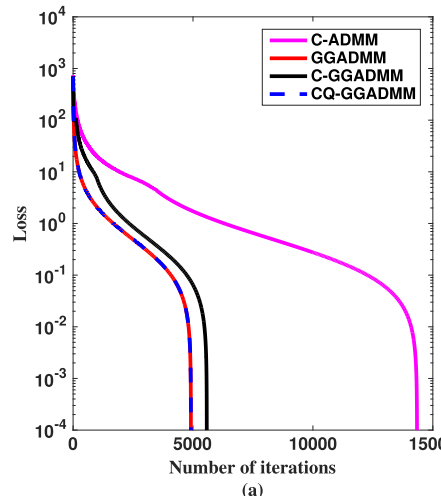

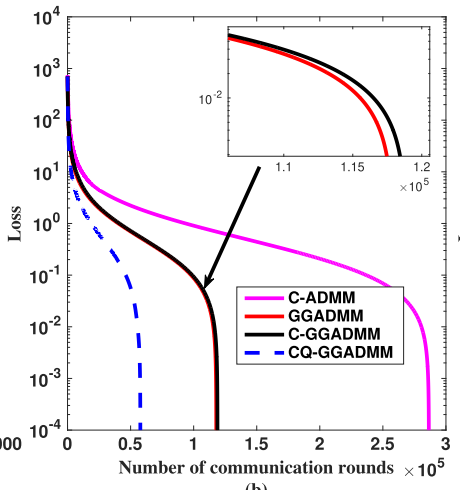

(b)

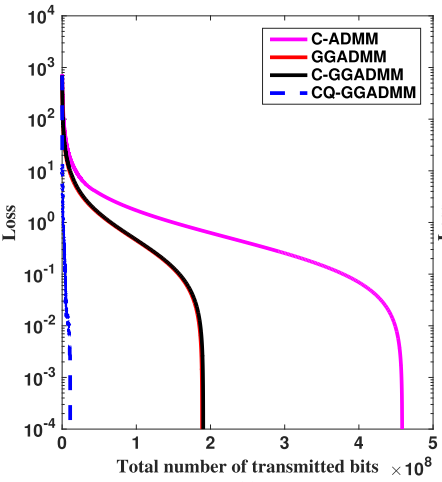

(c)

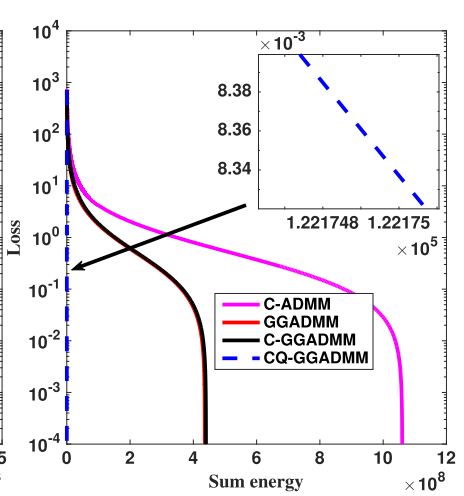

(d)

Fig. 4. Logistic regression results on synthetic dataset showing the loss w.r.t.: (a) \# iterations; (b) \# communication rounds; (c) \# transmitted bits; (d) total energy.

an experiment using linear regression on real dataset under different graph topologies. In particular, we consider two graphs with different density as shown in Fig. 6. The first graph, denoted by Graph 1, is a sparse graph (generated with $p=0.2$ ), where each worker has a few links (communicating with low number of neighbouring workers). For example, worker 12 communicates only with one neighbour (worker 8). On the other hand, the dense graph (Graph 2) is generated 


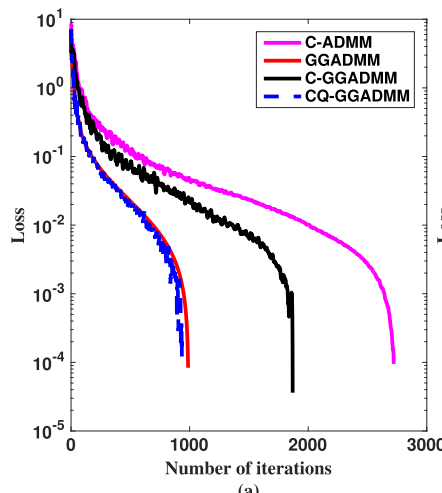

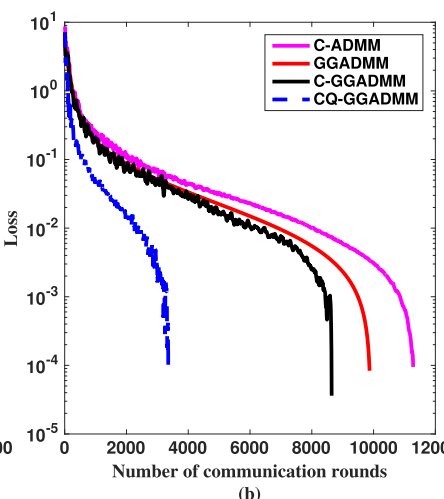

(b)

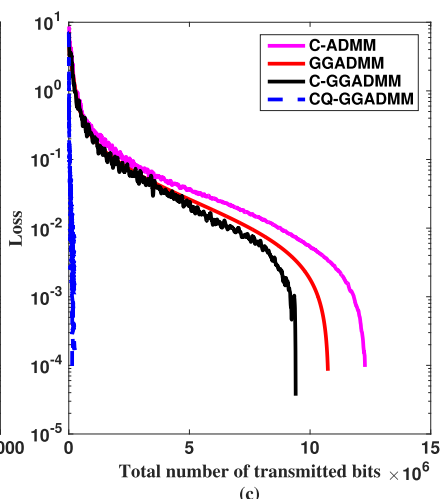

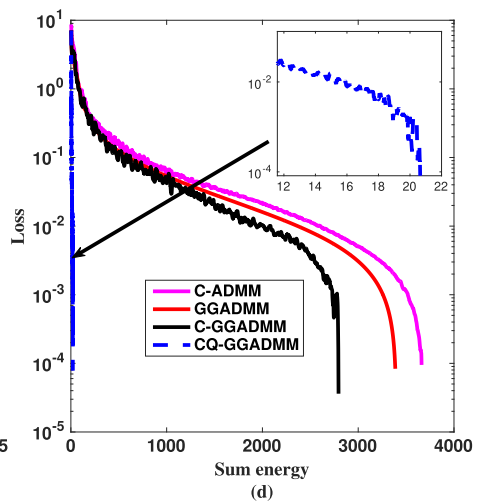

(d)

Fig. 5. Logistic regression results on real dataset showing the loss w.r.t.: (a) \# iterations; (b) \# communication rounds; (c) \# transmitted bits; (d) total energy.
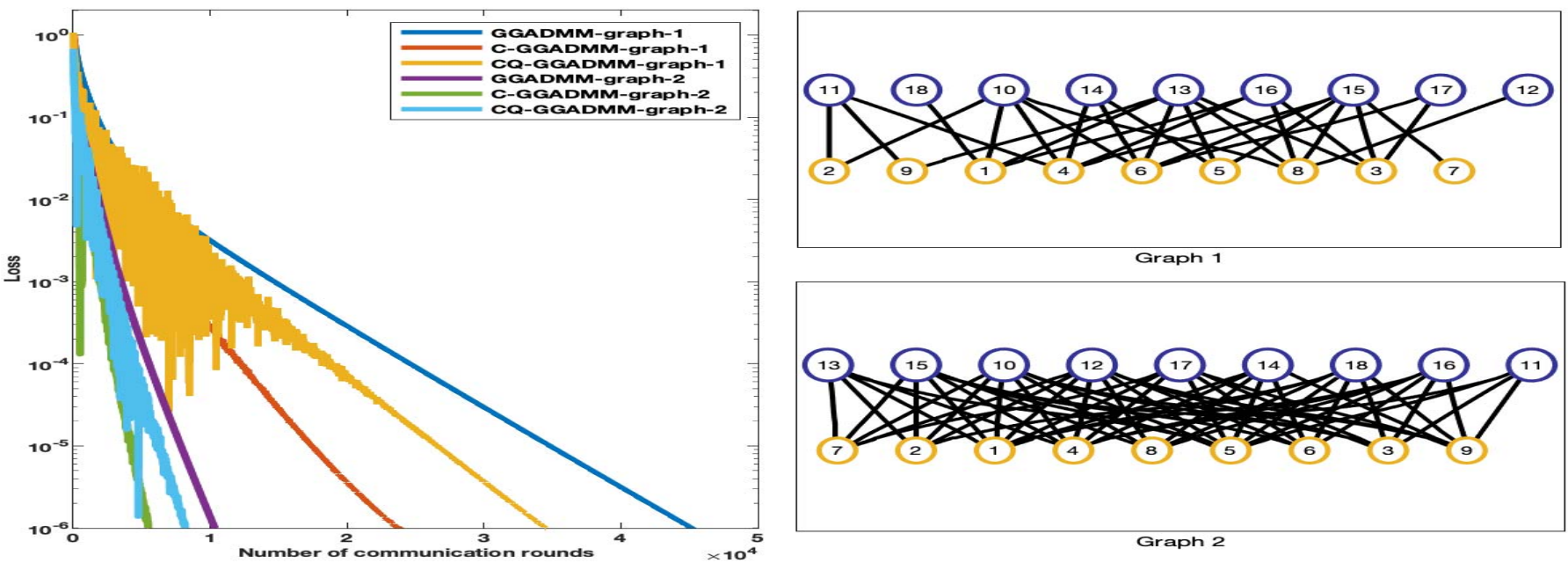

Fig. 6. Effect of the graph density on the performance of the algorithms: the loss w.r.t. \# communication rounds (left), Graph 1: Sparse graph (right top); Graph 2: dense graph (right bottom).
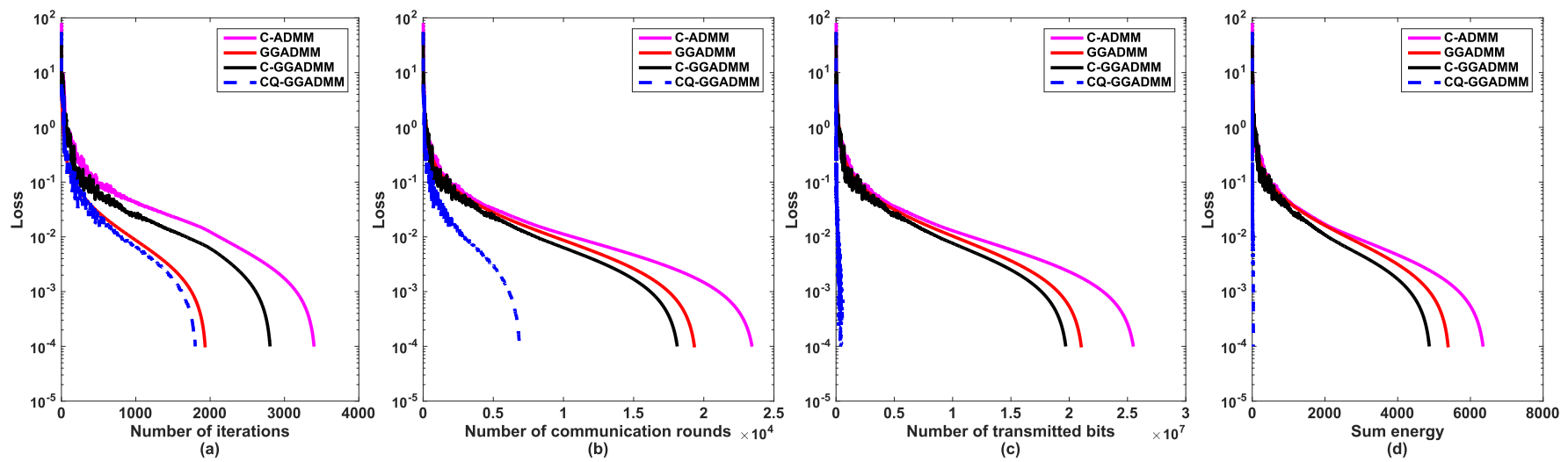

Fig. 7. Effect of the non-IIDness on the performance of the algorithms: the loss w.r.t.: (a) \# iterations; (b) \# communication rounds; (c) \# transmitted bits; (d) total energy.

with a connectivity ratio $p=0.4$ where each worker has at least three links (three neighbours). We can see from Fig. 6 that a denser graph leads to faster convergence for all algorithms since each worker uses more information per iteration. However, the ratio in the performance gap in terms of the number of communication rounds remains the same, i.e., C-GGADMM achieves the minimum number of communication rounds followed by CQ-GGADMM which confirms the findings in Fig.3-(b) for more choices of network graph density. 


\section{G. Impact of the Non-IIDness}

To study the impact of non-IIDness on the convergence of the different algorithms, we artificially create a non-IID partitioned data from the Derm dataset by first sorting the data by label and then dividing them between the workers. In Fig. 7, we study the effect of the non-IIDness on the performance of the different algorithms by plotting the loss as a function of the number of iterations, communications rounds, transmitted bits, and sum energy. Although it is clear that the non-IIDness of the data hurts the performance of all algorithms, CQ-GGADMM still provides significant savings in terms of communication rounds, and especially in transmitted bits and sum energy compared to the other baselines. Since the distribution of each local dataset differs from the global distribution, each worker's local objective can be different. As a result, the local updates can drift from the global optima. Hence, the averaged model may also be far from the global optimal, especially when the difference between the local updates is significant, i.e. the degree of heterogeneity between the local datasets is high. Consequently, the global model has significantly lower performance in the non-IID setting than the IID one.

\section{CONCLUSION}

In this paper, we have proposed a communication-efficiently decentralized ML algorithm that extends GADMM and Q-GADMM to arbitrary topologies. Moreover, the proposed algorithm leverages censoring (sparsification) to minimize the number of communication rounds for each worker. Utilizing a decreasing sequence of censoring threshold, stochastic quantization, and adjusting the quantization range at every iteration such that a linear convergence rate is achieved are key features that make CQ-GGADMM robust to errors while ensuring its convergence guarantees. Numerical results in convex linear and logistic regression tasks corroborate the advantages of CQ-GGADMM over GGADMM, and C-ADMM. An interesting direction is to consider solving the distributed learning problem over a time-varying topology. Additionally, extending the derivations to the stochastic non-convex setting is of paramount importance especially when training deep neural networks. Finally, improving model training on non-IID data is a key challenge that need to be addressed.

\section{APPENDICES}

\section{A. Basic Identities and Inequalities}

For any two vectors $\boldsymbol{x}, \boldsymbol{y} \in \mathbb{R}^{d}$, we have

$$
\begin{aligned}
\|\boldsymbol{x}+\boldsymbol{y}\|^{2} & \leq 2\left(\|\boldsymbol{x}\|^{2}+\|\boldsymbol{y}\|^{2}\right), \quad \forall \boldsymbol{x}, \boldsymbol{y} \in \mathbb{R}^{d}, \\
2\langle\boldsymbol{x}, \boldsymbol{y}\rangle & \leq \frac{1}{\eta}\|\boldsymbol{x}\|^{2}+\eta\|\boldsymbol{y}\|^{2}, \quad \forall \boldsymbol{x}, \boldsymbol{y} \in \mathbb{R}^{d}, \eta>0,
\end{aligned}
$$

For any two matrices $\boldsymbol{A}$ and $\boldsymbol{B}$, we have

$$
\begin{aligned}
2\langle\boldsymbol{A}, \boldsymbol{B}\rangle & \leq \eta\|\boldsymbol{A}\|_{F}^{2}+\frac{1}{\eta}\|\boldsymbol{B}\|_{F}^{2}, \quad \forall \eta>0, \\
\|\boldsymbol{A} \boldsymbol{B}\|_{F} & \leq \sigma_{\max }(\boldsymbol{A})\|\boldsymbol{B}\|_{F}, \\
\|\boldsymbol{A}+\boldsymbol{B}\|_{F}^{2} & \leq \eta\|\boldsymbol{A}\|_{F}^{2}+\frac{\eta}{\eta-1}\|\boldsymbol{B}\|_{F}^{2}, \quad \forall \eta>1,
\end{aligned}
$$

where $\sigma_{\max }(\boldsymbol{A})$ denotes the maximum singular value of the matrix $A$.

\section{B. Proof of Lemma 1}

Using (15) the update of the head workers can be written as

$$
\nabla f_{n}\left(\boldsymbol{\theta}_{n}^{k+1}\right)+\boldsymbol{\alpha}_{n}^{k}-\rho \sum_{m \in \mathcal{N}_{n}} \hat{\boldsymbol{\theta}}_{m}^{k}+\rho d_{n} \boldsymbol{\theta}_{n}^{k+1}=\mathbf{0} .
$$

Using the update of $\boldsymbol{\alpha}_{n}^{k}$ in (17), and the definition of the dual residual, we get

$$
\nabla f_{n}\left(\boldsymbol{\theta}_{n}^{k+1}\right)+\boldsymbol{\alpha}_{n}^{k+1}+\rho d_{n} \boldsymbol{\epsilon}_{n}^{k+1}+\boldsymbol{s}_{n}^{k+1}=\mathbf{0} .
$$

Thus, $\boldsymbol{\theta}_{n}^{k+1}$ minimizes the function $f_{n}\left(\boldsymbol{\theta}_{n}\right)+\left\langle\boldsymbol{\alpha}_{n}^{k+1}+\right.$ $\left.\rho d_{n} \boldsymbol{\epsilon}_{n}^{k+1}+\boldsymbol{s}_{n}^{k+1}, \boldsymbol{\theta}_{n}\right\rangle$ and as a consequence

$$
\begin{aligned}
& \mathbb{E}\left[f_{n}\left(\boldsymbol{\theta}_{n}^{k+1}\right)+\left\langle\boldsymbol{\alpha}_{n}^{k+1}+\rho d_{n} \boldsymbol{\epsilon}_{n}^{k+1}+\boldsymbol{s}_{n}^{k+1}, \boldsymbol{\theta}_{n}^{k+1}\right\rangle\right] \\
& \leq \mathbb{E}\left[f_{n}\left(\boldsymbol{\theta}_{n}^{\star}\right)+\left\langle\boldsymbol{\alpha}_{n}^{k+1}+\rho d_{n} \boldsymbol{\epsilon}_{n}^{k+1}+\boldsymbol{s}_{n}^{k+1}, \boldsymbol{\theta}_{n}^{\star}\right\rangle\right] .
\end{aligned}
$$

Similarly, using the update of the tail workers as in (16), we can write

$$
\nabla f_{m}\left(\boldsymbol{\theta}_{m}^{k+1}\right)+\boldsymbol{\alpha}_{m}^{k}-\rho \sum_{n \in \mathcal{N}_{m}} \hat{\boldsymbol{\theta}}_{n}^{k+1}+\rho d_{m} \boldsymbol{\theta}_{m}^{k+1}=\mathbf{0} .
$$

Hence, we get $\nabla f_{m}\left(\boldsymbol{\theta}_{m}^{k+1}\right)+\boldsymbol{\alpha}_{m}^{k+1}+\rho d_{m} \boldsymbol{\epsilon}_{m}^{k+1}=\mathbf{0}$. Thus, we can observe that the dual feasibility condition is fulfilled by the tail workers and $\boldsymbol{\theta}_{m}^{k+1}$ minimizes $f_{m}\left(\boldsymbol{\theta}_{m}\right)+\left\langle\boldsymbol{\alpha}_{m}^{k+1}+\right.$ $\left.\rho d_{m} \boldsymbol{\epsilon}_{m}^{k+1}, \boldsymbol{\theta}_{m}\right\rangle$. Therefore, we obtain the following inequality

$$
\begin{aligned}
& \mathbb{E}\left[f_{m}\left(\boldsymbol{\theta}_{m}^{k+1}\right)\right]+\mathbb{E}\left[\left\langle\boldsymbol{\alpha}_{m}^{k+1}+\rho d_{m} \boldsymbol{\epsilon}_{m}^{k+1}, \boldsymbol{\theta}_{m}^{k+1}\right\rangle\right] \\
& \leq \mathbb{E}\left[f_{m}\left(\boldsymbol{\theta}_{m}^{\star}\right)\right]+\mathbb{E}\left[\left\langle\boldsymbol{\alpha}_{m}^{k+1}+\rho d_{m} \boldsymbol{\epsilon}_{m}^{k+1}, \boldsymbol{\theta}_{m}^{\star}\right\rangle\right] .
\end{aligned}
$$

Summing over all workers, we get

$$
\begin{aligned}
& \sum_{n=1}^{N} \mathbb{E}\left[f_{n}\left(\boldsymbol{\theta}_{n}^{k+1}\right)-f_{n}\left(\boldsymbol{\theta}_{n}^{\star}\right)\right] \\
& \leq \sum_{n \in \mathcal{H}} \mathbb{E}\left[\left\langle\boldsymbol{\alpha}_{n}^{k+1}+\boldsymbol{s}_{n}^{k+1}+\rho d_{n} \boldsymbol{\epsilon}_{n}^{k+1}, \boldsymbol{\theta}_{n}^{\star}-\boldsymbol{\theta}_{n}^{k+1}\right\rangle\right] \\
& \quad+\sum_{m \in \mathcal{T}} \mathbb{E}\left[\left\langle\boldsymbol{\alpha}_{m}^{k+1}+\rho d_{m} \boldsymbol{\epsilon}_{m}^{k+1}, \boldsymbol{\theta}_{m}^{\star}-\boldsymbol{\theta}_{m}^{k+1}\right\rangle\right] \\
& \quad+\rho \sum_{n=1}^{N} \mathbb{E}\left[\left\langle d_{n} \boldsymbol{\epsilon}_{n}^{k+1}, \boldsymbol{\theta}_{n}^{\star}-\boldsymbol{\theta}_{n}^{k+1}\right\rangle\right] .
\end{aligned}
$$

Using the definition of $\boldsymbol{\alpha}_{n}^{k+1}, n \in \mathcal{V}$ in the right hand-side of (40)

$$
\begin{aligned}
& \sum_{n \in \mathcal{H}} \mathbb{E}\left[\left\langle\boldsymbol{\alpha}_{n}^{k+1}, \boldsymbol{\theta}_{n}^{\star}-\boldsymbol{\theta}_{n}^{k+1}\right\rangle\right]+\sum_{m \in \mathcal{T}} \mathbb{E}\left[\left\langle\boldsymbol{\alpha}_{m}^{k+1}, \boldsymbol{\theta}_{m}^{\star}-\boldsymbol{\theta}_{m}^{k+1}\right\rangle\right] \\
& =\sum_{(n, m) \in \mathcal{E}} \mathbb{E}\left[\left\langle\boldsymbol{\lambda}_{n, m}^{k+1}, \boldsymbol{\theta}_{n}^{\star}-\boldsymbol{\theta}_{n}^{k+1}\right\rangle\right] \\
& \quad+\sum_{(n, m) \in \mathcal{E}} \mathbb{E}\left[\left\langle\boldsymbol{\lambda}_{m, n}^{k+1}, \boldsymbol{\theta}_{m}^{\star}-\boldsymbol{\theta}_{m}^{k+1}\right\rangle\right] .
\end{aligned}
$$

Using that $\boldsymbol{\lambda}_{m, n}^{k+1}=-\boldsymbol{\lambda}_{n, m}^{k+1}$, and that $\boldsymbol{\theta}_{n}^{\star}=\boldsymbol{\theta}_{m}^{\star}, \forall(n, m) \in$ $\mathcal{E}$ we can write

$$
\begin{gathered}
\sum_{n \in \mathcal{H}} \mathbb{E}\left[\left\langle\boldsymbol{\alpha}_{n}^{k+1}, \boldsymbol{\theta}_{n}^{\star}-\boldsymbol{\theta}_{n}^{k+1}\right\rangle\right]+\sum_{m \in \mathcal{T}} \mathbb{E}\left[\left\langle\boldsymbol{\alpha}_{m}^{k+1}, \boldsymbol{\theta}_{m}^{\star}-\boldsymbol{\theta}_{m}^{k+1}\right\rangle\right] \\
=-\sum_{(n, m) \in \mathcal{E}} \mathbb{E}\left[\left\langle\boldsymbol{\lambda}_{n, m}^{k+1}, \boldsymbol{r}_{n, m}^{k+1}\right\rangle\right]
\end{gathered}
$$


This proves $(i)$ of Lemma 1 . To prove $(i i)$, we know from the optimality conditions that $\nabla f_{n}\left(\boldsymbol{\theta}_{n}^{\star}\right)+\boldsymbol{\alpha}_{n}^{\star}=\mathbf{0}$. Thus, $\boldsymbol{\theta}_{n}^{\star}$ minimizes the function $f_{n}\left(\boldsymbol{\theta}_{n}\right)+\left\langle\boldsymbol{\alpha}_{n}^{\star}, \boldsymbol{\theta}_{n}\right\rangle$ and for $n \in \mathcal{H}$

$\mathbb{E}\left[f_{n}\left(\boldsymbol{\theta}_{n}^{\star}\right)\right]+\mathbb{E}\left[\left\langle\boldsymbol{\alpha}_{n}^{\star}, \boldsymbol{\theta}_{n}^{\star}\right\rangle\right] \leq \mathbb{E}\left[f_{n}\left(\boldsymbol{\theta}_{n}^{k+1}\right)\right]+\mathbb{E}\left[\left\langle\boldsymbol{\alpha}_{n}^{\star}, \boldsymbol{\theta}_{n}^{k+1}\right\rangle\right]$.

Similarly, we have, for $m \in \mathcal{T}$, that

$$
\begin{aligned}
& \mathbb{E}\left[f_{m}\left(\boldsymbol{\theta}_{m}^{\star}\right)\right]+\mathbb{E}\left[\left\langle\boldsymbol{\alpha}_{m}^{\star}, \boldsymbol{\theta}_{m}^{\star}\right\rangle\right] \leq \mathbb{E}[\left.f_{m}\left(\boldsymbol{\theta}_{m}^{k+1}\right)\right] \\
&+\mathbb{E}\left[\left\langle\boldsymbol{\alpha}_{m}^{\star}, \boldsymbol{\theta}_{m}^{k+1}\right\rangle\right] .
\end{aligned}
$$

Summing over all workers, we get

$$
\begin{aligned}
\sum_{n=1}^{N} \mathbb{E}[ & \left.f_{n}\left(\boldsymbol{\theta}_{n}^{k+1}\right)-f_{n}\left(\boldsymbol{\theta}_{n}^{\star}\right)\right] \\
\geq & \sum_{n \in \mathcal{H}} \mathbb{E}\left[\left\langle\boldsymbol{\alpha}_{n}^{k+1}, \boldsymbol{\theta}_{n}^{\star}-\boldsymbol{\theta}_{n}^{k+1}\right\rangle\right] \\
& +\sum_{m \in \mathcal{T}} \mathbb{E}\left[\left\langle\boldsymbol{\alpha}_{m}^{k+1}, \boldsymbol{\theta}_{m}^{\star}-\boldsymbol{\theta}_{m}^{k+1}\right\rangle\right] \\
& \stackrel{\text { (a) }}{\geq} \sum_{(n, m) \in \mathcal{E}} \mathbb{E}\left[\left\langle\boldsymbol{\lambda}_{n, m}^{\star}, \boldsymbol{\theta}_{n}^{\star}-\boldsymbol{\theta}_{n}^{k+1}\right\rangle\right] \\
& +\sum_{(n, m) \in \mathcal{E}} \mathbb{E}\left[\left\langle\boldsymbol{\lambda}_{m, n}^{\star}, \boldsymbol{\theta}_{m}^{\star}-\boldsymbol{\theta}_{m}^{k+1}\right\rangle\right] \\
& (\text { (b) } \\
\geq & -\sum_{(n, m) \in \mathcal{E}} \mathbb{E}\left[\left\langle\boldsymbol{\lambda}_{n, m}^{\star}, \boldsymbol{r}_{n, m}^{k+1}\right\rangle\right],
\end{aligned}
$$

where we used the definition of $\boldsymbol{\alpha}_{n}^{\star}$ in (a) and that $\boldsymbol{\lambda}_{m, n}^{k+1}=-\boldsymbol{\lambda}_{n, m}^{k+1}$, and that $\boldsymbol{\theta}_{n}^{\star}=\boldsymbol{\theta}_{m}^{\star}$ in (b).

\section{Proof of Theorem 1}

Multiplying (26) by (-1), adding (25) and multiplying the sum by 2 , we get

$$
\begin{aligned}
& 2 \sum_{(n, m) \in \mathcal{E}} \mathbb{E}\left[\left\langle\boldsymbol{\lambda}_{n, m}^{\star}-\boldsymbol{\lambda}_{n, m}^{k+1}, \boldsymbol{r}_{n, m}^{k+1}\right\rangle\right] \\
& \quad+2 \sum_{n \in \mathcal{H}} \mathbb{E}\left[\left\langle\boldsymbol{s}_{n}^{k+1}, \boldsymbol{\theta}_{n}^{\star}-\boldsymbol{\theta}_{n}^{k+1}\right\rangle\right] \\
& \quad+2 \rho \sum_{n=1}^{N} \mathbb{E}\left[\left\langle d_{n} \boldsymbol{\epsilon}_{n}^{k+1}, \boldsymbol{\theta}_{n}^{\star}-\boldsymbol{\theta}_{n}^{k+1}\right\rangle\right] \geq 0 .
\end{aligned}
$$

Since $\boldsymbol{\lambda}_{n, m}^{k+1}=\boldsymbol{\lambda}_{n, m}^{k}+\rho \boldsymbol{r}_{n, m}^{k+1}+\rho\left(\boldsymbol{\epsilon}_{m}^{k+1}-\boldsymbol{\epsilon}_{n}^{k+1}\right)$, then we can write

$$
\begin{aligned}
2 & \sum_{(n, m) \in \mathcal{E}} \mathbb{E}\left[\left\langle\boldsymbol{\lambda}_{n, m}^{\star}-\boldsymbol{\lambda}_{n, m}^{k+1}, \boldsymbol{r}_{n, m}^{k+1}\right\rangle\right] \\
= & 2 \sum_{(n, m) \in \mathcal{E}} \mathbb{E}\left[\left\langle\boldsymbol{\lambda}_{n, m}^{\star}-\boldsymbol{\lambda}_{n, m}^{k}, \boldsymbol{r}_{n, m}^{k+1}\right\rangle\right]-2 \rho \sum_{(n, m) \in \mathcal{E}} \mathbb{E}\left[\left\|\boldsymbol{r}_{n, m}^{k+1}\right\|^{2}\right] \\
& -2 \rho \sum_{(n, m) \in \mathcal{E}} \mathbb{E}\left[\left\langle\boldsymbol{\epsilon}_{m}^{k+1}-\boldsymbol{\epsilon}_{n}^{k+1}, \boldsymbol{r}_{n, m}^{k+1}\right\rangle\right] .
\end{aligned}
$$

Using $\boldsymbol{r}_{n, m}^{k+1}=\frac{1}{\rho}\left(\boldsymbol{\lambda}_{n, m}^{k+1}-\boldsymbol{\lambda}_{n, m}^{\star}\right)-\frac{1}{\rho}\left(\boldsymbol{\lambda}_{n, m}^{k}-\boldsymbol{\lambda}_{n, m}^{\star}\right)+\boldsymbol{\epsilon}_{n}^{k+1}-$ $\epsilon_{m}^{k+1}$, we will examine the different terms of (47) starting from the first term

$$
\begin{aligned}
2 & \sum_{(n, m) \in \mathcal{E}} \mathbb{E}\left[\left\langle\boldsymbol{\lambda}_{n, m}^{\star}-\boldsymbol{\lambda}_{n, m}^{k}, \boldsymbol{r}_{n, m}^{k+1}\right\rangle\right] \\
= & \frac{2}{\rho} \sum_{(n, m) \in \mathcal{E}} \mathbb{E}\left[\left\langle\boldsymbol{\lambda}_{n, m}^{\star}-\boldsymbol{\lambda}_{n, m}^{k}, \boldsymbol{\lambda}_{n, m}^{k+1}-\boldsymbol{\lambda}_{n, m}^{\star}\right\rangle\right] \\
& +\frac{2}{\rho} \sum_{(n, m) \in \mathcal{E}} \mathbb{E}\left[\left\|\boldsymbol{\lambda}_{n, m}^{k}-\boldsymbol{\lambda}_{n, m}^{\star}\right\|^{2}\right] \\
& +2 \sum_{(n, m) \in \mathcal{E}} \mathbb{E}\left[\left\langle\boldsymbol{\lambda}_{n, m}^{\star}-\boldsymbol{\lambda}_{n, m}^{k}, \boldsymbol{\epsilon}_{n}^{k+1}-\boldsymbol{\epsilon}_{m}^{k+1}\right\rangle\right] .
\end{aligned}
$$

The second term can be re-written as

$$
\begin{aligned}
- & 2 \rho \sum_{(n, m) \in \mathcal{E}} \mathbb{E}\left[\left\|\boldsymbol{r}_{n, m}^{k+1}\right\|^{2}\right] \\
= & -\rho \sum_{(n, m) \in \mathcal{E}} \mathbb{E}\left[\left\|\boldsymbol{r}_{n, m}^{k+1}\right\|^{2}\right]-\frac{1}{\rho} \sum_{(n, m) \in \mathcal{E}} \mathbb{E}\left[\left\|\boldsymbol{\lambda}_{n, m}^{k+1}-\boldsymbol{\lambda}_{n, m}^{\star}\right\|^{2}\right] \\
& -\frac{1}{\rho} \sum_{(n, m) \in \mathcal{E}} \mathbb{E}\left[\left\|\boldsymbol{\lambda}_{n, m}^{k}-\boldsymbol{\lambda}_{n, m}^{\star}\right\|^{2}\right] \\
& -\rho \sum_{(n, m) \in \mathcal{E}} \mathbb{E}\left[\left\|\boldsymbol{\epsilon}_{n}^{k+1}-\boldsymbol{\epsilon}_{m}^{k+1}\right\|^{2}\right] \\
& +\frac{2}{\rho} \sum_{(n, m) \in \mathcal{E}} \mathbb{E}\left[\left\langle\boldsymbol{\lambda}_{n, m}^{k+1}-\boldsymbol{\lambda}_{n, m}^{\star}, \boldsymbol{\lambda}_{n, m}^{k}-\boldsymbol{\lambda}_{n, m}^{\star}\right\rangle\right] \\
& -2 \sum_{(n, m) \in \mathcal{E}} \mathbb{E}\left[\left\langle\boldsymbol{\lambda}_{n, m}^{k+1}-\boldsymbol{\lambda}_{n, m}^{\star}, \boldsymbol{\epsilon}_{n}^{k+1}-\boldsymbol{\epsilon}_{m}^{k+1}\right\rangle\right] \\
& +2 \sum_{(n, m) \in \mathcal{E}} \mathbb{E}\left[\left\langle\boldsymbol{\lambda}_{n, m}^{k}-\boldsymbol{\lambda}_{n, m}^{\star}, \boldsymbol{\epsilon}_{n}^{k+1}-\boldsymbol{\epsilon}_{m}^{k+1}\right\rangle\right]
\end{aligned}
$$

The third term can be expanded as

$$
\begin{aligned}
-2 \rho \sum_{(n, m) \in \mathcal{E}} \mathbb{E}\left[\left\langle\boldsymbol{\epsilon}_{m}^{k+1}-\boldsymbol{\epsilon}_{n}^{k+1}, \boldsymbol{r}_{n, m}^{k+1}\right\rangle\right] \\
=-2 \sum_{(n, m) \in \mathcal{E}} \mathbb{E}\left[\left\langle\boldsymbol{\epsilon}_{m}^{k+1}-\boldsymbol{\epsilon}_{n}^{k+1}, \boldsymbol{\lambda}_{n, m}^{k+1}-\boldsymbol{\lambda}_{n, m}^{\star}\right\rangle\right] \\
\quad+2 \sum_{(n, m) \in \mathcal{E}} \mathbb{E}\left[\left\langle\boldsymbol{\epsilon}_{m}^{k+1}-\boldsymbol{\epsilon}_{n}^{k+1}, \boldsymbol{\lambda}_{n, m}^{k}-\boldsymbol{\lambda}_{n, m}^{\star}\right\rangle\right] \\
\quad+2 \rho \sum_{(n, m) \in \mathcal{E}} \mathbb{E}\left[\left\|\boldsymbol{\epsilon}_{n}^{k+1}-\boldsymbol{\epsilon}_{m}^{k+1}\right\|^{2}\right] .
\end{aligned}
$$

From Eqs. (48)-(50), we can re-write (47) as

$$
\begin{aligned}
& 2 \sum_{(n, m) \in \mathcal{E}} \mathbb{E}\left[\left\langle\boldsymbol{\lambda}_{n, m}^{\star}-\boldsymbol{\lambda}_{n, m}^{k+1}, \boldsymbol{r}_{n, m}^{k+1}\right\rangle\right] \\
&= \frac{1}{\rho} \sum_{(n, m) \in \mathcal{E}} \mathbb{E}\left[\left\|\boldsymbol{\lambda}_{n, m}^{k}-\boldsymbol{\lambda}_{n, m}^{\star}\right\|^{2}\right] \\
&-\frac{1}{\rho} \sum_{(n, m) \in \mathcal{E}} \mathbb{E}\left[\left\|\boldsymbol{\lambda}_{n, m}^{k+1}-\boldsymbol{\lambda}_{n, m}^{\star}\right\|^{2}\right] \\
&-\rho \sum_{(n, m) \in \mathcal{E}} \mathbb{E}\left[\left\|\boldsymbol{r}_{n, m}^{k+1}\right\|^{2}\right] \\
&+2 \sum_{(n, m) \in \mathcal{E}} \mathbb{E}\left[\left\langle\boldsymbol{\epsilon}_{m}^{k+1}-\boldsymbol{\epsilon}_{n}^{k+1}, \boldsymbol{\lambda}_{n, m}^{k}-\boldsymbol{\lambda}_{n, m}^{\star}\right\rangle\right] \\
&+\rho \sum_{(n, m) \in \mathcal{E}} \mathbb{E}\left[\left\|\boldsymbol{\epsilon}_{n}^{k+1}-\boldsymbol{\epsilon}_{m}^{k+1}\right\|^{2}\right] .
\end{aligned}
$$


The second term of the left hand-side of (46) can be decomposed as

$$
\begin{array}{rl}
2 \sum_{n \in \mathcal{H}} & \mathbb{E}\left[\left\langle\boldsymbol{s}_{n}^{k+1}, \boldsymbol{\theta}_{n}^{\star}-\boldsymbol{\theta}_{n}^{k+1}\right\rangle\right] \\
= & 2 \rho \sum_{(n, m) \in \mathcal{E}} \mathbb{E}\left[\left\langle\hat{\boldsymbol{\theta}}_{m}^{k+1}-\hat{\boldsymbol{\theta}}_{m}^{k}, \boldsymbol{\theta}_{n}^{\star}-\boldsymbol{\theta}_{m}^{k+1}\right\rangle\right] \\
& -2 \rho \sum_{(n, m) \in \mathcal{E}} \mathbb{E}\left[\left\langle\hat{\boldsymbol{\theta}}_{m}^{k+1}-\hat{\boldsymbol{\theta}}_{m}^{k}, \boldsymbol{r}_{n, m}^{k+1}\right\rangle\right] .
\end{array}
$$

Now, we can re-write the first term as

$$
\begin{aligned}
-2 \rho & \sum_{(n, m) \in \mathcal{E}} \mathbb{E}\left[\left\langle\hat{\boldsymbol{\theta}}_{m}^{k+1}-\hat{\boldsymbol{\theta}}_{m}^{k}, \boldsymbol{r}_{n, m}^{k+1}\right\rangle\right] \\
= & -2 \rho \sum_{(n, m) \in \mathcal{E}} \mathbb{E}\left[\left\langle\boldsymbol{\theta}_{m}^{k+1}-\boldsymbol{\theta}_{m}^{k}, \boldsymbol{r}_{n, m}^{k+1}\right\rangle\right] \\
& +2 \rho \sum_{(n, m) \in \mathcal{E}} \mathbb{E}\left[\left\langle\boldsymbol{\epsilon}_{m}^{k+1}-\boldsymbol{\epsilon}_{m}^{k}, \boldsymbol{r}_{n, m}^{k+1}\right\rangle\right] .
\end{aligned}
$$

The second term can be expanded as

$$
\begin{aligned}
& 2 \rho \sum_{(n, m) \in \mathcal{E}} \mathbb{E}\left[\left\langle\hat{\boldsymbol{\theta}}_{m}^{k+1}-\hat{\boldsymbol{\theta}}_{m}^{k}, \boldsymbol{\theta}_{n}^{\star}-\boldsymbol{\theta}_{m}^{k+1}\right\rangle\right] \\
& =2 \rho \sum_{(n, m) \in \mathcal{E}} \mathbb{E}\left[\left\langle\boldsymbol{\theta}_{m}^{k+1}-\boldsymbol{\theta}_{m}^{k}, \boldsymbol{\theta}_{n}^{\star}-\boldsymbol{\theta}_{m}^{k+1}\right\rangle\right] \\
& \quad+2 \rho \sum_{(n, m) \in \mathcal{E}} \mathbb{E}\left[\left\langle\boldsymbol{\epsilon}_{m}^{k+1}-\boldsymbol{\epsilon}_{m}^{k}, \boldsymbol{\theta}_{m}^{k+1}-\boldsymbol{\theta}_{n}^{\star}\right\rangle\right] .
\end{aligned}
$$

Since $\boldsymbol{\theta}_{n}^{*}=\boldsymbol{\theta}_{m}^{*}, \forall(n, m) \in \mathcal{E}$ and $\boldsymbol{\theta}_{m}^{\star}-\boldsymbol{\theta}_{m}^{k+1}=\boldsymbol{\theta}_{m}^{\star}-\boldsymbol{\theta}_{m}^{k}+$ $\boldsymbol{\theta}_{m}^{k}-\boldsymbol{\theta}_{m}^{k+1}$, we can write

$$
\begin{aligned}
2 \rho & \sum_{(n, m) \in \mathcal{E}} \mathbb{E}\left[\left\langle\boldsymbol{\theta}_{m}^{k+1}-\boldsymbol{\theta}_{m}^{k}, \boldsymbol{\theta}_{n}^{\star}-\boldsymbol{\theta}_{m}^{k+1}\right\rangle\right] \\
= & -\rho \sum_{(n, m) \in \mathcal{E}} \mathbb{E}\left[\left\|\boldsymbol{\theta}_{m}^{k+1}-\boldsymbol{\theta}_{m}^{k}\right\|^{2}\right] \\
& -\rho \sum_{(n, m) \in \mathcal{E}} \mathbb{E}\left[\left\|\boldsymbol{\theta}_{m}^{k+1}-\boldsymbol{\theta}_{m}^{\star}\right\|^{2}\right] \\
& -\rho \sum_{(n, m) \in \mathcal{E}} \mathbb{E}\left[\left\|\boldsymbol{\theta}_{m}^{k}-\boldsymbol{\theta}_{m}^{\star}\right\|^{2}\right] \\
& +2 \rho \sum_{(n, m) \in \mathcal{E}} \mathbb{E}\left[\left\langle\boldsymbol{\theta}_{m}^{k+1}-\boldsymbol{\theta}_{m}^{\star}, \boldsymbol{\theta}_{m}^{k}-\boldsymbol{\theta}_{m}^{\star}\right\rangle\right] .
\end{aligned}
$$

With this expression at hand, we can go back to (52)

$$
\begin{aligned}
2 \sum_{n \in \mathcal{H}} \mathbb{E} & {\left[\left\langle\boldsymbol{s}_{n}^{k+1}, \boldsymbol{\theta}_{n}^{\star}-\boldsymbol{\theta}_{n}^{k+1}\right\rangle\right] } \\
= & \rho \sum_{(n, m) \in \mathcal{E}} \mathbb{E}\left[\left\|\boldsymbol{\theta}_{m}^{k}-\boldsymbol{\theta}_{m}^{\star}\right\|^{2}\right] \\
& -\rho \sum_{(n, m) \in \mathcal{E}} \mathbb{E}\left[\left\|\boldsymbol{\theta}_{m}^{k+1}-\boldsymbol{\theta}_{m}^{\star}\right\|^{2}\right] \\
& +2 \rho \sum_{(n, m) \in \mathcal{E}} \mathbb{E}\left[\left\langle\boldsymbol{\epsilon}_{m}^{k+1}-\boldsymbol{\epsilon}_{m}^{k}, \boldsymbol{\theta}_{m}^{k+1}-\boldsymbol{\theta}_{n}^{\star}\right\rangle\right] \\
& -2 \rho \sum_{(n, m) \in \mathcal{E}} \mathbb{E}\left[\left\langle\boldsymbol{\theta}_{m}^{k+1}-\boldsymbol{\theta}_{m}^{k}, \boldsymbol{r}_{n, m}^{k+1}\right\rangle\right]
\end{aligned}
$$

$$
\begin{aligned}
& +2 \rho \sum_{(n, m) \in \mathcal{E}} \mathbb{E}\left[\left\langle\boldsymbol{\epsilon}_{m}^{k+1}-\boldsymbol{\epsilon}_{m}^{k}, \boldsymbol{r}_{n, m}^{k+1}\right\rangle\right] \\
& -\rho \sum_{(n, m) \in \mathcal{E}} \mathbb{E}\left[\left\|\boldsymbol{\theta}_{m}^{k+1}-\boldsymbol{\theta}_{m}^{k}\right\|^{2}\right] .
\end{aligned}
$$

Replacing (51) and (56) in (46), we obtain

$$
\begin{aligned}
\frac{1}{\rho} \sum_{(n, m) \in \mathcal{E}} \mathbb{E}\left[\left\|\boldsymbol{\lambda}_{n, m}^{k}-\boldsymbol{\lambda}_{n, m}^{\star}\right\|^{2}\right] & \frac{1}{\rho} \sum_{(n, m) \in \mathcal{E}} \mathbb{E}\left[\left\|\boldsymbol{\lambda}_{n, m}^{k+1}-\boldsymbol{\lambda}_{n, m}^{\star}\right\|^{2}\right]-\rho \sum_{(n, m) \in \mathcal{E}} \mathbb{E}\left[\left\|\boldsymbol{r}_{n, m}^{k+1}\right\|^{2}\right] \\
& +2 \sum_{(n, m) \in \mathcal{E}} \mathbb{E}\left[\left\langle\boldsymbol{\epsilon}_{m}^{k+1}-\boldsymbol{\epsilon}_{n}^{k+1}, \boldsymbol{\lambda}_{n, m}^{k}-\boldsymbol{\lambda}_{n, m}^{\star}\right\rangle\right] \\
& +\rho \sum_{(n, m) \in \mathcal{E}} \mathbb{E}\left[\left\|\boldsymbol{\epsilon}_{n}^{k+1}-\boldsymbol{\epsilon}_{m}^{k+1}\right\|^{2}\right]+\rho \sum_{(n, m) \in \mathcal{E}} \mathbb{E}\left[\left\|\boldsymbol{\theta}_{m}^{k}-\boldsymbol{\theta}_{m}^{\star}\right\|^{2}\right] \\
& -\rho \sum_{(n, m) \in \mathcal{E}} \mathbb{E}\left[\left\|\boldsymbol{\theta}_{m}^{k+1}-\boldsymbol{\theta}_{m}^{\star}\right\|^{2}\right]-\rho \sum_{(n, m) \in \mathcal{E}} \mathbb{E}\left[\left\|\boldsymbol{\theta}_{m}^{k+1}-\boldsymbol{\theta}_{m}^{k}\right\|^{2}\right] \\
& -2 \rho \sum_{(n, m) \in \mathcal{E}} \mathbb{E}\left[\left\langle\boldsymbol{\theta}_{m}^{k+1}-\boldsymbol{\theta}_{m}^{k}, \boldsymbol{r}_{n, m}^{k+1}\right\rangle\right] \\
& +2 \rho \sum_{(n, m) \in \mathcal{E}} \mathbb{E}\left[\left\langle\boldsymbol{\epsilon}_{m}^{k+1}-\boldsymbol{\epsilon}_{m}^{k}, \boldsymbol{r}_{n, m}^{k+1}\right\rangle\right] \\
& +2 \rho \sum_{(n, m) \in \mathcal{E}} \mathbb{E}\left[\left\langle\boldsymbol{\epsilon}_{m}^{k+1}-\boldsymbol{\epsilon}_{m}^{k}, \boldsymbol{\theta}_{m}^{k+1}-\boldsymbol{\theta}_{n}^{\star}\right\rangle\right] \\
& +2 \rho \sum_{n=1}^{N} \mathbb{E}\left[\left\langle d_{n} \boldsymbol{\epsilon}_{n}^{k+1}, \boldsymbol{\theta}_{n}^{\star}-\boldsymbol{\theta}_{n}^{k+1}\right\rangle\right] \geq 0 .
\end{aligned}
$$

Using the identity $\boldsymbol{r}_{n, m}^{k+1}=\frac{1}{\rho}\left(\boldsymbol{\lambda}_{n, m}^{k+1}-\boldsymbol{\lambda}_{n, m}^{k}\right)+\boldsymbol{\epsilon}_{n}^{k+1}-\boldsymbol{\epsilon}_{m}^{k+1}$, we can write

$$
\begin{aligned}
& -\rho \sum_{(n, m) \in \mathcal{E}} \mathbb{E}\left[\left\|\boldsymbol{r}_{n, m}^{k+1}\right\|^{2}\right]=-\frac{1}{\rho} \sum_{(n, m) \in \mathcal{E}} \mathbb{E}\left[\left\|\boldsymbol{\lambda}_{n, m}^{k+1}-\boldsymbol{\lambda}_{n, m}^{k}\right\|^{2}\right] \\
& -\rho \sum_{(n, m) \in \mathcal{E}} \mathbb{E}\left[\left\|\boldsymbol{\epsilon}_{n}^{k+1}-\boldsymbol{\epsilon}_{m}^{k+1}\right\|^{2}\right] \\
& +2 \sum_{(n, m) \in \mathcal{E}} \mathbb{E}\left[\left\langle\boldsymbol{\lambda}_{n, m}^{k+1}-\boldsymbol{\lambda}_{n, m}^{k}, \boldsymbol{\epsilon}_{m}^{k+1}-\boldsymbol{\epsilon}_{n}^{k+1}\right\rangle\right]
\end{aligned}
$$

On the other hand, we have

$$
\begin{aligned}
& 2 \rho \sum_{(n, m) \in \mathcal{E}} \mathbb{E}\left[\left\langle\boldsymbol{\epsilon}_{m}^{k+1}-\boldsymbol{\epsilon}_{m}^{k}, \boldsymbol{r}_{n, m}^{k+1}\right\rangle\right] \\
& \quad+2 \rho \sum_{n=1}^{N} \mathbb{E}\left[\left\langle d_{n} \boldsymbol{\epsilon}_{n}^{k+1}, \boldsymbol{\theta}_{n}^{\star}-\boldsymbol{\theta}_{n}^{k+1}\right\rangle\right] \\
& =2 \rho \sum_{(n, m) \in \mathcal{E}} \mathbb{E}\left[\left\langle\boldsymbol{\epsilon}_{n}^{k+1}+\boldsymbol{\epsilon}_{m}^{k+1}, \boldsymbol{\theta}_{m}^{\star}-\boldsymbol{\theta}_{m}^{k+1}\right\rangle\right] \\
& \quad-2 \rho \sum_{(n, m) \in \mathcal{E}} \mathbb{E}\left[\left\|\boldsymbol{\epsilon}_{n}^{k+1}-\boldsymbol{\epsilon}_{m}^{k+1}\right\|^{2}\right]-2 \rho \sum_{(n, m) \in \mathcal{E}} \mathbb{E}\left[\left\langle\boldsymbol{\epsilon}_{m}^{k}, \boldsymbol{r}_{n, m}^{k+1}\right\rangle\right] \\
& \quad+2 \rho \sum_{(n, m) \in \mathcal{E}} \mathbb{E}\left[\left\langle\boldsymbol{\lambda}_{n, m}^{k+1}-\boldsymbol{\lambda}_{n, m}^{k}, \boldsymbol{\epsilon}_{m}^{k+1}-\boldsymbol{\epsilon}_{n}^{k+1}\right\rangle\right] .
\end{aligned}
$$

Now, recall that $\boldsymbol{\theta}_{m}^{k+1}, m \in \mathcal{T}$ minimizes the function $f_{m}\left(\boldsymbol{\theta}_{m}\right)+\left\langle\boldsymbol{\alpha}_{m}^{k+1}+\rho d_{m} \boldsymbol{\epsilon}_{m}^{k+1}, \boldsymbol{\theta}_{m}\right\rangle$ and $\boldsymbol{\theta}_{m}^{k}, m \in \mathcal{T}$ minimizes the function $f_{m}\left(\boldsymbol{\theta}_{m}\right)+\left\langle\boldsymbol{\alpha}_{m}^{k}+\rho d_{m} \boldsymbol{\epsilon}_{m}^{k}, \boldsymbol{\theta}_{m}\right\rangle$, then we could 
write

$$
\begin{array}{r}
\mathbb{E}\left[f_{m}\left(\boldsymbol{\theta}_{m}^{k+1}\right)\right]+\mathbb{E}\left[\left\langle\boldsymbol{\alpha}_{m}^{k+1}+\rho d_{m} \boldsymbol{\epsilon}_{m}^{k+1}, \boldsymbol{\theta}_{m}^{k+1}\right\rangle\right] \\
\leq \mathbb{E}\left[f_{m}\left(\boldsymbol{\theta}_{m}^{k}\right)\right]+\mathbb{E}\left[\left\langle\boldsymbol{\alpha}_{m}^{k+1}+\rho d_{m} \boldsymbol{\epsilon}_{m}^{k+1}, \boldsymbol{\theta}_{m}^{k}\right\rangle\right], \\
\mathbb{E}\left[f_{m}\left(\boldsymbol{\theta}_{m}^{k}\right)\right]+\mathbb{E}\left[\left\langle\boldsymbol{\alpha}_{m}^{k}+\rho d_{m} \boldsymbol{\epsilon}_{m}^{k}, \boldsymbol{\theta}_{m}^{k}\right\rangle\right] \\
\quad \leq \mathbb{E}\left[f_{m}\left(\boldsymbol{\theta}_{m}^{k+1}\right)\right]+\mathbb{E}\left[\left\langle\boldsymbol{\alpha}_{m}^{k}+\rho d_{m} \boldsymbol{\epsilon}_{m}^{k}, \boldsymbol{\theta}_{m}^{k+1}\right\rangle\right] .
\end{array}
$$

Adding both equations and re-arranging the terms, we get

$$
\begin{aligned}
\mathbb{E}\left[\left\langle\boldsymbol{\alpha}_{m}^{k+1}-\boldsymbol{\alpha}_{m}^{k}, \boldsymbol{\theta}_{m}^{k+1}-\boldsymbol{\theta}_{m}^{k}\right\rangle\right] \\
\leq-\rho d_{m} \mathbb{E}\left[\left\langle\boldsymbol{\epsilon}_{m}^{k+1}-\boldsymbol{\epsilon}_{m}^{k}, \boldsymbol{\theta}_{m}^{k+1}-\boldsymbol{\theta}_{m}^{k}\right\rangle\right] .
\end{aligned}
$$

Using the update of $\boldsymbol{\alpha}_{m}^{k+1}$, i.e. $\boldsymbol{\alpha}_{m}^{k+1}=\boldsymbol{\alpha}_{m}^{k}+$ $\rho \sum_{n \in \mathcal{N}_{m}} \boldsymbol{r}_{m, n}^{k+1}$, we can re-write (62) to get

$$
\begin{aligned}
-\rho \sum_{(n, m) \in \mathcal{E}} \mathbb{E}\left[\left\langle\boldsymbol{r}_{n, m}^{k+1}, \boldsymbol{\theta}_{m}^{k+1}-\boldsymbol{\theta}_{m}^{k}\right\rangle\right] \\
\quad \leq-\rho \sum_{(n, m) \in \mathcal{E}} \mathbb{E}\left[\left\langle\boldsymbol{\epsilon}_{m}^{k+1}-\boldsymbol{\epsilon}_{m}^{k}, \boldsymbol{\theta}_{m}^{k+1}-\boldsymbol{\theta}_{m}^{k}\right\rangle\right] .
\end{aligned}
$$

where we used $\boldsymbol{r}_{m, n}^{k+1}=-\boldsymbol{r}_{n, m}^{k+1}$ after summing over $m \in \mathcal{T}$. Going back to (57), we can write

$$
\begin{aligned}
& \frac{1}{\rho} \sum_{(n, m) \in \mathcal{E}} \mathbb{E}\left[\left\|\boldsymbol{\lambda}_{n, m}^{k+1}-\boldsymbol{\lambda}_{n, m}^{\star}\right\|^{2}\right]-\frac{1}{\rho} \sum_{(n, m) \in \mathcal{E}} \mathbb{E}\left[\left\|\boldsymbol{\lambda}_{n, m}^{k}-\boldsymbol{\lambda}_{n, m}^{\star}\right\|^{2}\right] \\
& \quad+\frac{1}{\rho} \sum_{(n, m) \in \mathcal{E}} \mathbb{E}\left[\left\|\boldsymbol{\lambda}_{n, m}^{k+1}-\boldsymbol{\lambda}_{n, m}^{k}\right\|^{2}\right] \\
& \quad+\rho \sum_{(n, m) \in \mathcal{E}} \mathbb{E}\left[\left\|\boldsymbol{\theta}_{m}^{k+1}-\boldsymbol{\theta}_{m}^{\star}\right\|^{2}\right] \\
& \quad-\rho \sum_{(n, m) \in \mathcal{E}} \mathbb{E}\left[\left\|\boldsymbol{\theta}_{m}^{k}-\boldsymbol{\theta}_{m}^{\star}\right\|^{2}\right]+\rho \sum_{(n, m) \in \mathcal{E}} \mathbb{E}\left[\left\|\boldsymbol{\theta}_{m}^{k+1}-\boldsymbol{\theta}_{m}^{k}\right\|^{2}\right] \\
& \leq 2 \sum_{(n, m) \in \mathcal{E}} \mathbb{E}\left[\left\langle\boldsymbol{\lambda}_{n, m}^{k+1}-\boldsymbol{\lambda}_{n, m}^{\star}, \boldsymbol{\epsilon}_{m}^{k+1}-\boldsymbol{\epsilon}_{n}^{k+1}\right\rangle\right] \\
& \quad+2 \sum_{(n, m) \in \mathcal{E}} \mathbb{E}\left[\left\langle\boldsymbol{\lambda}_{n, m}^{k+1}-\boldsymbol{\lambda}_{n, m}^{k}, \boldsymbol{\epsilon}_{m}^{k+1}-\boldsymbol{\epsilon}_{n}^{k+1}\right\rangle\right] \\
& \quad-2 \rho \sum_{(n, m) \in \mathcal{E}} \mathbb{E}\left[\left\langle\boldsymbol{\theta}_{m}^{k+1}-\boldsymbol{\theta}_{m}^{k}, \boldsymbol{\epsilon}_{m}^{k+1}-\boldsymbol{\epsilon}_{m}^{k}\right\rangle\right] \\
& \quad-2 \rho \sum_{(n, m) \in \mathcal{E}} \mathbb{E}\left[\left\langle\boldsymbol{\epsilon}_{m}^{k}, \boldsymbol{r}_{n, m}^{k+1}\right\rangle\right] \\
& \quad+2 \rho \sum_{(n, m) \in \mathcal{E}} \mathbb{E}\left[\left\langle\boldsymbol{\epsilon}_{m}^{k}+\boldsymbol{\epsilon}_{n}^{k+1}, \boldsymbol{\theta}_{m}^{\star}-\boldsymbol{\theta}_{m}^{k+1}\right\rangle\right] .
\end{aligned}
$$

To upper bound the terms in the right hand side, we will use the identity (31)

$$
\begin{aligned}
& 2 \sum_{(n, m) \in \mathcal{E}} \mathbb{E}\left[\left\langle\boldsymbol{\lambda}_{n, m}^{k+1}-\boldsymbol{\lambda}_{n, m}^{\star}, \boldsymbol{\epsilon}_{m}^{k+1}-\boldsymbol{\epsilon}_{n}^{k+1}\right\rangle\right] \\
& \leq \frac{1}{\eta_{1}} \sum_{(n, m) \in \mathcal{E}} \mathbb{E}\left[\left\|\boldsymbol{\epsilon}_{m}^{k+1}-\boldsymbol{\epsilon}_{n}^{k+1}\right\|^{2}\right] \\
&+\eta_{1} \sum_{(n, m) \in \mathcal{E}} \mathbb{E}\left[\left\|\boldsymbol{\lambda}_{n, m}^{k+1}-\boldsymbol{\lambda}_{n, m}^{\star}\right\|^{2}\right]
\end{aligned}
$$

$$
\begin{array}{r}
2 \sum_{(n, m) \in \mathcal{E}} \mathbb{E}\left[\left\langle\boldsymbol{\lambda}_{n, m}^{k+1}-\boldsymbol{\lambda}_{n, m}^{k}, \boldsymbol{\epsilon}_{m}^{k+1}-\boldsymbol{\epsilon}_{n}^{k+1}\right\rangle\right] \\
\leq \frac{1}{\eta_{2}} \sum_{(n, m) \in \mathcal{E}} \mathbb{E}\left[\left\|\boldsymbol{\epsilon}_{m}^{k+1}-\boldsymbol{\epsilon}_{n}^{k+1}\right\|^{2}\right] \\
+\eta_{2} \sum_{(n, m) \in \mathcal{E}} \mathbb{E}\left[\left\|\boldsymbol{\lambda}_{n, m}^{k+1}-\boldsymbol{\lambda}_{n, m}^{k}\right\|^{2}\right], \\
2 \rho \sum_{(n, m) \in \mathcal{E}} \mathbb{E}\left[\left\langle\boldsymbol{\epsilon}_{m}^{k}+\boldsymbol{\epsilon}_{n}^{k+1}, \boldsymbol{\theta}_{m}^{\star}-\boldsymbol{\theta}_{m}^{k+1}\right\rangle\right] \\
\leq \frac{\rho}{\eta_{3}} \sum_{(n, m) \in \mathcal{E}} \mathbb{E}\left[\left\|\boldsymbol{\epsilon}_{m}^{k}+\boldsymbol{\epsilon}_{n}^{k+1}\right\|^{2}\right] \\
+\rho \eta_{3} \sum_{(n, m) \in \mathcal{E}} \mathbb{E}\left[\left\|\boldsymbol{\theta}_{m}^{\star}-\boldsymbol{\theta}_{m}^{k+1}\right\|^{2}\right], \\
-2 \rho \sum_{(n, m) \in \mathcal{E}} \mathbb{E}\left[\left\langle\boldsymbol{\theta}_{m}^{k+1}-\boldsymbol{\theta}_{m}^{k}, \boldsymbol{\epsilon}_{m}^{k+1}-\boldsymbol{\epsilon}_{m}^{k}\right\rangle\right] \\
\leq \frac{\rho}{\eta_{4}} \sum_{(n, m) \in \mathcal{E}} \mathbb{E}\left[\left\|\boldsymbol{\epsilon}_{m}^{k+1}-\boldsymbol{\epsilon}_{m}^{k}\right\|^{2}\right] \\
\quad+\rho \eta_{4} \sum_{(n, m) \in \mathcal{E}} \mathbb{E}\left[\left\|\boldsymbol{\theta}_{m}^{k+1}-\boldsymbol{\theta}_{m}^{k}\right\|^{2}\right],
\end{array}
$$

Finally, we use both identities (30) and (31) to get the following bound

$$
\begin{aligned}
& -2 \rho \sum_{(n, m) \in \mathcal{E}} \mathbb{E}\left[\left\langle\boldsymbol{\epsilon}_{m}^{k}, \boldsymbol{r}_{n, m}^{k+1}\right\rangle\right] \\
& \leq \frac{\rho}{\eta_{5}} \sum_{(n, m) \in \mathcal{E}} \mathbb{E}\left[\left\|\boldsymbol{\epsilon}_{m}^{k}\right\|^{2}\right]+\frac{2 \rho}{\eta_{5}} \sum_{(n, m) \in \mathcal{E}} \mathbb{E}\left[\left\|\boldsymbol{\lambda}_{n, m}^{k+1}-\boldsymbol{\lambda}_{n, m}^{k}\right\|^{2}\right] \\
& \quad+2 \rho \eta_{5} \sum_{(n, m) \in \mathcal{E}} \mathbb{E}\left[\left\|\boldsymbol{\epsilon}_{m}^{k+1}-\boldsymbol{\epsilon}_{n}^{k+1}\right\|^{2}\right],
\end{aligned}
$$

where $\left\{\eta_{i}\right\}_{i=1}^{5}$ are arbitrary positive constants to be specified later on. Using these bounds and re-arranging the terms in (64), we can write

$$
\begin{aligned}
& 2 \rho \sum_{(n, m) \in \mathcal{E}} \mathbb{E}\left[\left\|\boldsymbol{\epsilon}_{n}^{k+1}-\boldsymbol{\epsilon}_{m}^{k+1}\right\|^{2}\right] \\
&+\rho\left(1-\eta_{4}\right) \sum_{(n, m) \in \mathcal{E}} \mathbb{E}\left[\left\|\boldsymbol{\theta}_{m}^{k+1}-\boldsymbol{\theta}_{m}^{k}\right\|^{2}\right] \\
& \quad+\left(\frac{1-2 \eta_{5}}{\rho}-\eta_{2}\right) \sum_{(n, m) \in \mathcal{E}} \mathbb{E}\left[\left\|\boldsymbol{\lambda}_{n, m}^{k+1}-\boldsymbol{\lambda}_{n, m}^{k}\right\|^{2}\right] \\
& \leq\left(\frac{1}{\eta_{1}}+\frac{1}{\eta_{2}}+2 \rho \eta_{5}\right) \sum_{(n, m) \in \mathcal{E}} \mathbb{E}\left[\left\|\boldsymbol{\epsilon}_{n}^{k+1}-\boldsymbol{\epsilon}_{m}^{k+1}\right\|^{2}\right] \\
&+\frac{\rho}{\eta_{3}} \sum_{(n, m) \in \mathcal{E}} \mathbb{E}\left[\left\|\boldsymbol{\epsilon}_{n}^{k+1}+\boldsymbol{\epsilon}_{m}^{k}\right\|^{2}\right] \\
&+\frac{1}{\rho} \sum_{(n, m) \in \mathcal{E}} \mathbb{E}\left[\left\|\boldsymbol{\lambda}_{n, m}^{k}-\boldsymbol{\lambda}_{n, m}^{\star}\right\|^{2}\right] \\
&-\left(\frac{1}{\rho}-\eta_{1}\right) \sum_{(n, m) \in \mathcal{E}} \mathbb{E}\left[\left\|\boldsymbol{\lambda}_{n, m}^{k+1}-\boldsymbol{\lambda}_{n, m}^{\star}\right\|^{2}\right] \\
&+\rho \sum_{(n, m) \in \mathcal{E}} \mathbb{E}\left[\left\|\boldsymbol{\theta}_{m}^{k}-\boldsymbol{\theta}_{m}^{\star}\right\|^{2}\right] \\
&-\rho\left(1-\eta_{3}\right) \sum_{(n, m) \in \mathcal{E}} \mathbb{E}\left[\left\|\boldsymbol{\theta}_{m}^{k+1}-\boldsymbol{\theta}_{m}^{\star}\right\|^{2}\right]
\end{aligned}
$$




$$
\begin{aligned}
& +\frac{\rho}{\eta_{5}} \sum_{(n, m) \in \mathcal{E}} \mathbb{E}\left[\left\|\boldsymbol{\epsilon}_{m}^{k}\right\|^{2}\right] \\
& +\frac{\rho}{\eta_{4}} \sum_{(n, m) \in \mathcal{E}} \mathbb{E}\left[\left\|\boldsymbol{\epsilon}_{m}^{k+1}-\boldsymbol{\epsilon}_{m}^{k}\right\|^{2}\right] .
\end{aligned}
$$

Fix the values of $\left\{\eta_{i}\right\}_{i=1}^{5}$ to be $\left(\eta_{1}, \eta_{2}, \eta_{3}, \eta_{4}, \eta_{5}\right)=$ $\left(\frac{\psi^{k}}{2 \psi^{0} \rho}, \frac{1}{4 \rho}, \frac{\psi^{k}}{2 \psi^{0}}, \frac{1}{2}, \frac{1}{4}\right)$, we get

$$
\begin{aligned}
\frac{\rho}{2} & \sum_{(n, m) \in \mathcal{E}} \mathbb{E}\left[\left\|\boldsymbol{\theta}_{m}^{k+1}-\boldsymbol{\theta}_{m}^{k}\right\|^{2}\right] \\
& +\frac{1}{4 \rho} \sum_{(n, m) \in \mathcal{E}} \mathbb{E}\left[\left\|\boldsymbol{\lambda}_{n, m}^{k+1}-\boldsymbol{\lambda}_{n, m}^{k}\right\|^{2}\right] \\
\leq & \left(\frac{5 \rho}{2}+\frac{2 \rho \psi^{0}}{\psi^{k}}\right) \sum_{(n, m) \in \mathcal{E}} \mathbb{E}\left[\left\|\boldsymbol{\epsilon}_{n}^{k+1}-\boldsymbol{\epsilon}_{m}^{k+1}\right\|^{2}\right] \\
& +\frac{2 \rho \psi^{0}}{\psi^{k}} \sum_{(n, m) \in \mathcal{E}} \mathbb{E}\left[\left\|\boldsymbol{\epsilon}_{n}^{k+1}+\boldsymbol{\epsilon}_{m}^{k}\right\|^{2}\right] \\
& +\frac{1}{\rho} \sum_{(n, m) \in \mathcal{E}} \mathbb{E}\left[\left\|\boldsymbol{\lambda}_{n, m}^{k}-\boldsymbol{\lambda}_{n, m}^{\star}\right\|^{2}\right] \\
& -\frac{1}{\rho}\left(1-\frac{\psi^{k}}{2 \psi^{0}}\right) \sum_{(n, m) \in \mathcal{E}} \mathbb{E}\left[\left\|\boldsymbol{\lambda}_{n, m}^{k+1}-\boldsymbol{\lambda}_{n, m}^{\star}\right\|^{2}\right] \\
& +\rho \sum_{(n, m) \in \mathcal{E}} \mathbb{E}\left[\left\|\boldsymbol{\theta}_{m}^{k}-\boldsymbol{\theta}_{m}^{\star}\right\|^{2}\right] \\
& -\rho\left(1-\frac{\psi^{k}}{2 \psi^{0}}\right) \sum_{(n, m) \in \mathcal{E}} \mathbb{E}\left[\left\|\boldsymbol{\theta}_{m}^{k+1}-\boldsymbol{\theta}_{m}^{\star}\right\|^{2}\right] \\
& +4 \rho \sum_{(n, m) \in \mathcal{E}} \mathbb{E}\left[\left\|\boldsymbol{\epsilon}_{m}^{k}\right\|^{2}\right]+2 \rho \sum_{(n, m) \in \mathcal{E}} \mathbb{E}\left[\left\|\boldsymbol{\epsilon}_{m}^{k+1}-\boldsymbol{\epsilon}_{m}^{k}\right\|^{2}\right]
\end{aligned}
$$

Re-arranging the terms, we can write

$$
\begin{aligned}
& \frac{\rho}{2} \sum_{(n, m) \in \mathcal{E}} \mathbb{E}\left[\left\|\boldsymbol{\theta}_{m}^{k+1}-\boldsymbol{\theta}_{m}^{k}\right\|^{2}\right] \\
&+\frac{1}{4 \rho} \sum_{(n, m) \in \mathcal{E}} \mathbb{E}\left[\left\|\boldsymbol{\lambda}_{n, m}^{k+1}-\boldsymbol{\lambda}_{n, m}^{k}\right\|^{2}\right] \\
& \leq \frac{1}{\rho} \sum_{(n, m) \in \mathcal{E}} \mathbb{E}\left[\left\|\boldsymbol{\lambda}_{n, m}^{k}-\boldsymbol{\lambda}_{n, m}^{\star}\right\|^{2}\right] \\
&-\frac{1}{\rho}\left(1-\frac{\psi^{k}}{2 \psi^{0}}\right) \mathbb{E}\left[\left\|\boldsymbol{\lambda}_{n, m}^{k+1}-\boldsymbol{\lambda}_{n, m}^{\star}\right\|^{2}\right] \\
&+\rho \sum_{(n, m) \in \mathcal{E}} \mathbb{E}\left[\left\|\boldsymbol{\theta}_{m}^{k}-\boldsymbol{\theta}_{m}^{\star}\right\|^{2}\right] \\
&-\rho\left(1-\frac{\psi^{k}}{2 \psi^{0}}\right) \sum_{(n, m) \in \mathcal{E}} \mathbb{E}\left[\left\|\boldsymbol{\theta}_{m}^{k+1}-\boldsymbol{\theta}_{m}^{\star}\right\|^{2}\right] \\
&+\left(5 \rho+\frac{8 \rho \psi^{0}}{\psi^{k}}\right) \sum_{(n, m) \in \mathcal{E}} \mathbb{E}\left[\left\|\boldsymbol{\epsilon}_{n}^{k+1}\right\|^{2}\right] \\
&+\left(9 \rho+\frac{4 \rho \psi^{0}}{\psi^{k}}\right) \sum_{(n, m) \in \mathcal{E}} \mathbb{E}\left[\left\|\boldsymbol{\epsilon}_{m}^{k+1}\right\|^{2}\right] \\
&+\left(8 \rho+\frac{4 \rho \psi^{0}}{\psi^{k}}\right) \sum_{(n, m) \in \mathcal{E}} \mathbb{E}\left[\left\|\boldsymbol{\epsilon}_{m}^{k}\right\|^{2}\right] .
\end{aligned}
$$

Therefore, using (24), we can write

$$
\begin{aligned}
\frac{\rho}{2} \sum_{(n, m) \in \mathcal{E}} \mathbb{E}\left[\left\|\boldsymbol{\theta}_{m}^{k+1}-\boldsymbol{\theta}_{m}^{k}\right\|^{2}\right] \\
\quad+\frac{1}{4 \rho} \sum_{(n, m) \in \mathcal{E}} \mathbb{E}\left[\left\|\boldsymbol{\lambda}_{n, m}^{k+1}-\boldsymbol{\lambda}_{n, m}^{k}\right\|^{2}\right] \\
\leq \frac{1}{\rho} \sum_{(n, m) \in \mathcal{E}} \mathbb{E}\left[\left\|\boldsymbol{\lambda}_{n, m}^{k}-\boldsymbol{\lambda}_{n, m}^{\star}\right\|^{2}\right] \\
\quad-\frac{1}{\rho}\left(1-\frac{\psi^{k}}{2 \psi^{0}}\right) \mathbb{E}\left[\left\|\boldsymbol{\lambda}_{n, m}^{k+1}-\boldsymbol{\lambda}_{n, m}^{\star}\right\|^{2}\right] \\
+\rho \sum_{(n, m) \in \mathcal{E}} \mathbb{E}\left[\left\|\boldsymbol{\theta}_{m}^{k}-\boldsymbol{\theta}_{m}^{\star}\right\|^{2}\right] \\
\quad-\rho\left(1-\frac{\psi^{k}}{2 \psi^{0}}\right) \sum_{(n, m) \in \mathcal{E}} \mathbb{E}\left[\left\|\boldsymbol{\theta}_{m}^{k+1}-\boldsymbol{\theta}_{m}^{\star}\right\|^{2}\right] \\
\quad+\gamma_{1} \psi^{k}+\gamma_{2} \psi^{2 k},
\end{aligned}
$$

where $\gamma_{1}=64 \rho C_{0} \psi^{0}|\mathcal{E}|$ and $\gamma_{2}=88 \rho C_{0}^{2}|\mathcal{E}|$. Now, we define the Lyapunov function

$V^{k}=\frac{1}{\rho} \sum_{(n, m) \in \mathcal{E}}\left\|\boldsymbol{\lambda}_{n, m}^{k}-\boldsymbol{\lambda}_{n, m}^{\star}\right\|^{2}+\rho \sum_{(n, m) \in \mathcal{E}}\left\|\boldsymbol{\theta}_{m}^{k}-\boldsymbol{\theta}_{m}^{\star}\right\|^{2}$.

Thus, we get

$$
\begin{gathered}
\frac{\rho}{2} \sum_{(n, m) \in \mathcal{E}} \mathbb{E}\left[\left\|\boldsymbol{\theta}_{m}^{k+1}-\boldsymbol{\theta}_{m}^{k}\right\|^{2}\right]+\frac{1}{4 \rho} \sum_{(n, m) \in \mathcal{E}} \mathbb{E}\left[\left\|\boldsymbol{\lambda}_{n, m}^{k+1}-\boldsymbol{\lambda}_{n, m}^{k}\right\|^{2}\right] \\
\leq \mathbb{E}\left[V^{k}\right]-\left(1-\frac{\psi^{k}}{2 \psi^{0}}\right) \mathbb{E}\left[V^{k+1}\right]+\gamma_{1} \psi^{k}+\gamma_{2} \psi^{2 k} .
\end{gathered}
$$

As a consequence, we can write that $\mathbb{E}\left[V^{k+1}\right] \leq$ $\left(1-\frac{\psi^{k}}{2 \psi^{0}}\right)^{-1}\left(\mathbb{E}\left[V^{k}\right]+\gamma_{1} \psi^{k}+\gamma_{2} \psi^{2 k}\right)$. Using this equation iteratively, we obtain

$$
\begin{aligned}
\mathbb{E} & {\left[V^{k+1}\right] } \\
\leq & \left(\prod_{j=0}^{k}\left(1-\frac{\psi^{j}}{2 \psi^{0}}\right)^{-1}\right) \mathbb{E}\left[V_{0}\right]+\gamma_{1} \sum_{j=0}^{k} \prod_{i=j}^{k}\left(1-\frac{\psi^{i}}{2 \psi^{0}}\right)^{-1} \psi^{j} \\
& +\gamma_{2} \sum_{j=0}^{k} \prod_{i=j}^{k}\left(1-\frac{\psi^{i}}{2 \psi^{0}}\right)^{-1} \psi^{2 j} \\
\leq & \prod_{j=0}^{\infty}\left(1-\frac{\psi^{j}}{2 \psi^{0}}\right)^{-1}\left(\mathbb{E}\left[V^{0}\right]+\gamma_{1} \sum_{i=0}^{\infty} \psi^{i}+\gamma_{2} \sum_{i=0}^{\infty} \psi^{2 i}\right) .
\end{aligned}
$$

where we have used the fact that $\left(1-\frac{\psi^{k}}{2 \psi^{0}}\right) \in\left[\frac{1}{2}, 1\right]$. Since $\sum_{i=0}^{\infty} \omega^{i}<\infty$ and $\sum_{i=0}^{\infty} \xi^{i}<\infty$, thus $\sum_{i=0}^{\infty} \psi^{i}<\infty$. Furthermore, the sequence $\left\{\psi^{i}\right\}$ is non-negative, then we get that $\sum_{i=0}^{\infty} \psi^{2 i}<\infty$. To show that $\prod_{j=0}^{\infty}\left(1-\frac{\psi^{j}}{2 \psi^{0}}\right)^{-1}$ is also finite, we consider its logarithm, i.e.

$$
\log \left(\prod_{j=0}^{\infty}\left(1-\frac{\psi^{j}}{2 \psi^{0}}\right)^{-1}\right) \stackrel{(\text { a) }}{\leq} \sum_{j=0}^{\infty} \log \left(\left(1-\frac{\psi^{j}}{2 \psi^{0}}\right)^{-1}\right)
$$




$$
\stackrel{\text { (b) }}{\leq} \sum_{j=0}^{\infty} \log \left(1+\frac{\psi^{j}}{\psi^{0}}\right) \leq \frac{1}{\psi^{0}} \sum_{j=0}^{\infty} \psi^{j} \text {, }
$$

where we have used that $\log \left(\left(1-\frac{z}{2}\right)^{-1}\right) \leq \log (1+z), z \geq$ 1 in (a) and $\log (1+z) \leq z, z \geq 1$ in (b). Hence, $\prod_{j=0}^{\infty}\left(1-\frac{\psi^{j}}{2 \psi^{0}}\right)^{-1}$ is also finite and we conclude that the sequence $\mathbb{E}\left[V^{k}\right]$ is upper bounded by a finite quantity that we denote as $\bar{V}$. Going back to (75) and taking the sum from $k=0$ to $\infty$ while using the upper bound on $\mathbb{E}\left[V^{k}\right]$, we get

$$
\begin{gathered}
\sum_{k=0}^{\infty} \sum_{(n, m) \in \mathcal{E}}\left\{\frac{\rho}{2} \mathbb{E}\left[\left\|\boldsymbol{\theta}_{m}^{k+1}-\boldsymbol{\theta}_{m}^{k}\right\|^{2}\right]+\frac{1}{4 \rho} \mathbb{E}\left[\left\|\boldsymbol{\lambda}_{n, m}^{k+1}-\boldsymbol{\lambda}_{n, m}^{k}\right\|^{2}\right]\right\} \\
\leq V^{0}+\left(\frac{\bar{V}}{2 \psi^{0}}+\gamma_{1}\right) \sum_{k=0}^{\infty} \psi^{k}+\gamma_{2} \sum_{k=0}^{\infty} \psi^{2 k} .
\end{gathered}
$$

Since the right hand side is finite, we conclude that the left hand side is convergent and that

$$
\begin{aligned}
& \lim _{k \rightarrow \infty} \mathbb{E}\left[\left\|\boldsymbol{\theta}_{m}^{k+1}-\boldsymbol{\theta}_{m}^{k}\right\|^{2}\right]=0, \\
& \lim _{k \rightarrow \infty} \mathbb{E}\left[\left\|\boldsymbol{\lambda}_{n, m}^{k+1}-\boldsymbol{\lambda}_{n, m}^{k}\right\|^{2}\right]=0 .
\end{aligned}
$$

Using the definition of the primal and dual residuals and (30), we can write

$$
\begin{aligned}
& \mathbb{E}\left[\left\|\boldsymbol{r}_{n, m}^{k+1}\right\|^{2}\right] \leq 2\left(\frac{1}{\rho^{2}} \mathbb{E}\left[\left\|\boldsymbol{\lambda}_{n, m}^{k+1}-\boldsymbol{\lambda}_{n, m}^{k}\right\|^{2}\right]\right. \\
&\left.+2\left(\mathbb{E}\left[\left\|\boldsymbol{\epsilon}_{m}^{k}\right\|^{2}\right]+\mathbb{E}\left[\left\|\boldsymbol{\epsilon}_{m}^{k+1}\right\|^{2}\right]\right)\right) \\
& \mathbb{E}\left[\left\|\boldsymbol{s}_{n}^{k+1}\right\|^{2}\right] \leq 2 \rho^{2} d_{n}\left(\sum_{m \in \mathcal{N}_{n}} \mathbb{E}\left[\left\|\boldsymbol{\theta}_{m}^{k+1}-\boldsymbol{\theta}_{m}^{k}\right\|^{2}\right]\right. \\
&\left.+\sum_{m \in \mathcal{N}_{n}} \mathbb{E}\left[\left\|\boldsymbol{\epsilon}_{m}^{k}-\boldsymbol{\epsilon}_{m}^{k+1}\right\|^{2}\right]\right) .
\end{aligned}
$$

Since $\mathbb{E}\left[\left\|\boldsymbol{\epsilon}_{n}^{k}\right\|^{2}\right] \leq 4 C_{0}^{2} \psi^{2 k}, \forall n$, then $\lim _{k \rightarrow \infty} \mathbb{E}\left[\left\|\boldsymbol{\epsilon}_{n}^{k}\right\|^{2}\right]=0$. Using (79), (80) and $\lim _{k \rightarrow \infty} \mathbb{E}\left[\left\|\epsilon_{n}^{k}\right\|^{2}\right]=0$, we conclude that $\lim _{k \rightarrow \infty} \mathbb{E}\left[\left\|\boldsymbol{r}_{n, m}^{k+1}\right\|^{2}\right]=0$ and $\lim _{k \rightarrow \infty} \mathbb{E}\left[\left\|\boldsymbol{s}_{n}^{k+1}\right\|^{2}\right]=0$.

$k \rightarrow \infty$
Using the Cauchy-Schwarz inequality, we can write

$$
\begin{aligned}
& \left|\mathbb{E}\left[\left\langle\boldsymbol{\lambda}_{n, m}^{k+1}, \boldsymbol{r}_{n, m}^{k+1}\right\rangle\right]\right|^{2} \leq \mathbb{E}\left[\left\|\boldsymbol{\lambda}_{n, m}^{k+1}\right\|^{2}\right] \mathbb{E}\left[\left\|\boldsymbol{r}_{n, m}^{k+1}\right\|^{2}\right] \\
& \left|\mathbb{E}\left[\left\langle\boldsymbol{\lambda}_{n, m}^{\star}, \boldsymbol{r}_{n, m}^{k+1}\right\rangle\right]\right|^{2} \leq \mathbb{E}\left[\left\|\boldsymbol{\lambda}_{n, m}^{\star}\right\|^{2}\right] \mathbb{E}\left[\left\|\boldsymbol{r}_{n, m}^{k+1}\right\|^{2}\right], \\
& \left|\mathbb{E}\left[\left\langle\boldsymbol{s}_{n}^{k+1}, \boldsymbol{\theta}_{n}^{\star}-\boldsymbol{\theta}_{n}^{k+1}\right\rangle\right]\right|^{2} \leq \mathbb{E}\left[\left\|\boldsymbol{s}_{n}^{k+1}\right\|^{2}\right] \mathbb{E}\left[\left\|\boldsymbol{\theta}_{n}^{\star}-\boldsymbol{\theta}_{n}^{k+1}\right\|^{2}\right],
\end{aligned}
$$

$\left|\mathbb{E}\left[\left\langle\boldsymbol{\epsilon}_{n}^{k+1}, \boldsymbol{\theta}_{n}^{\star}-\boldsymbol{\theta}_{n}^{k+1}\right\rangle\right]\right|^{2} \leq \mathbb{E}\left[\left\|\boldsymbol{\epsilon}_{n}^{k+1}\right\|^{2}\right] \mathbb{E}\left[\left\|\boldsymbol{\theta}_{n}^{\star}-\boldsymbol{\theta}_{n}^{k+1}\right\|^{2}\right]$.

Since $\lim _{k \rightarrow \infty} \mathbb{E}\left[\left\|\boldsymbol{\epsilon}_{n}^{k}\right\|^{2}\right]=0, \lim _{k \rightarrow \infty} \mathbb{E}\left[\left\|\boldsymbol{r}_{n, m}^{k+1}\right\|^{2}\right]=0$ and $\lim _{k \rightarrow \infty} \mathbb{E}\left[\left\|s_{n}^{k \rightarrow+\infty}\right\|^{2}\right]=0$, we get that

$$
\begin{aligned}
& \lim _{k \rightarrow \infty} \mathbb{E}\left[\left\langle\boldsymbol{\lambda}_{n, m}^{k+1}, \boldsymbol{r}_{n, m}^{k+1}\right\rangle\right]=0, \\
& \lim _{k \rightarrow \infty} \mathbb{E}\left[\left\langle\boldsymbol{\lambda}_{n, m}^{\star}, \boldsymbol{r}_{n, m}^{k+1}\right\rangle\right]=0,
\end{aligned}
$$

$$
\begin{aligned}
& \lim _{k \rightarrow \infty} \mathbb{E}\left[\left\langle\boldsymbol{s}_{n}^{k+1}, \boldsymbol{\theta}_{n}^{\star}-\boldsymbol{\theta}_{n}^{k+1}\right\rangle\right]=0, \\
& \lim _{k \rightarrow \infty} \mathbb{E}\left[\left\langle\boldsymbol{\epsilon}_{n}^{k+1}, \boldsymbol{\theta}_{n}^{\star}-\boldsymbol{\theta}_{n}^{k+1}\right\rangle\right]=0 .
\end{aligned}
$$

From $(i)$ and $(i i)$ of Lemma 1, we conclude that $\lim _{k \rightarrow \infty} \sum_{n=1}^{N} \mathbb{E}\left[f_{n}\left(\boldsymbol{\theta}_{n}^{k}\right)-f_{n}\left(\boldsymbol{\theta}_{n}^{\star}\right)\right]=0$.

\section{Proof of Theorem 2}

The proof of Theorem 2 follows similar steps as the proof of convergence rate of [13]. However, the alternating update nature of our algorithm makes the updates happen in an asymmetric manner, in contrast to the symmetric update in [13], which makes the proof more complex. For a bipartite graph, the adjacency matrix can be written as $\boldsymbol{A}=\left[\mathbf{0}_{r r}, \boldsymbol{B} ; \boldsymbol{B}^{T}, \mathbf{0}_{s s}\right]$ where $r=|\mathcal{H}|, s=|\mathcal{T}|$ are the cardinalities of $\mathcal{H}$ and $\mathcal{T}$, respectively. The matrices $\mathbf{0}_{r r}$, and $\mathbf{0}_{s s}$ are the null matrices of order $r \times r$, and $s \times s$, respectively. The matrix $\boldsymbol{B} \in \mathbb{R}^{r \times s}$ is called the bi-adjacency matrix. The adjacency matrix is a boolean matrix where each element is defined as $\boldsymbol{A}_{i, j}=1$ if there exists a link between the nodes $i$ and $j$ (i.e. workers), otherwise $\boldsymbol{A}_{i, j}=0$. In our analysis, we also define the matrix $\boldsymbol{C}=\left[\mathbf{0}_{r r}, \boldsymbol{B} ; \mathbf{0}_{r s}, \mathbf{0}_{s s}\right]$ as well as the matrices $\boldsymbol{\theta}=$ $\left[\boldsymbol{\theta}_{1}, \ldots, \boldsymbol{\theta}_{N}\right]^{T}, \boldsymbol{\alpha}=\left[\boldsymbol{\alpha}_{1}, \ldots, \boldsymbol{\alpha}_{N}\right]^{T}, \hat{\boldsymbol{\theta}}=\left[\hat{\boldsymbol{\theta}}_{1}, \ldots, \hat{\boldsymbol{\theta}}_{N}\right]^{T}$, and $\boldsymbol{E}=\left[\boldsymbol{\epsilon}_{1}, \ldots, \boldsymbol{\epsilon}_{N}\right]^{T}$. In this section, we introduce matrices related to the network topology: the diagonal degree matrix $\boldsymbol{D}$, the oriented and unoriented incidence matrices, $\boldsymbol{M}_{-}$and $M_{+}$, respectively. Using (17), (35), and (38), we can write

$$
\begin{aligned}
& \nabla f\left(\boldsymbol{\theta}^{k+1}\right)+\boldsymbol{\alpha}^{k}-\rho \boldsymbol{C} \hat{\boldsymbol{\theta}}^{k}-\rho \boldsymbol{C}^{T} \hat{\boldsymbol{\theta}}^{k+1}+\rho \boldsymbol{D} \boldsymbol{\theta}^{k+1}=\mathbf{0} \\
& \boldsymbol{\alpha}^{k+1}=\boldsymbol{\alpha}^{k}+\rho(\boldsymbol{D}-\boldsymbol{A}) \hat{\boldsymbol{\theta}}^{k+1}
\end{aligned}
$$

and the optimality conditions are given by $\nabla f\left(\boldsymbol{\theta}^{\star}\right)+\boldsymbol{\alpha}^{\star}=\mathbf{0}$, and $\boldsymbol{M}_{-}^{T} \boldsymbol{\theta}^{\star}=\mathbf{0}$. Since we have $\boldsymbol{D}-\boldsymbol{A}=\frac{1}{2} \boldsymbol{M}_{-} \boldsymbol{M}_{-}^{T}$, then we can re-write (92) as $\boldsymbol{\alpha}^{k+1}=\boldsymbol{\alpha}^{k}+\frac{\rho}{2} \boldsymbol{M}_{-} \boldsymbol{M}_{-}^{T} \boldsymbol{\theta}^{k+1}+$ $\frac{\rho}{2} \boldsymbol{M}_{-} \boldsymbol{M}_{-}^{T} \boldsymbol{E}^{k+1}$. Initializing $\boldsymbol{\alpha}^{0}$ in the column space of $\boldsymbol{M}_{-}$, we get that $\boldsymbol{\alpha}^{k}$ always stays in the column space of $\boldsymbol{M}_{-}$and thus, we have $\boldsymbol{\alpha}^{k}=\boldsymbol{M}_{-} \boldsymbol{\beta}^{k}, \forall k \geq 0$. Thus, we can further write (92) as

$$
\boldsymbol{\beta}^{k+1}=\boldsymbol{\beta}^{k}+\frac{\rho}{2} \boldsymbol{M}_{-}^{T} \boldsymbol{\theta}^{k+1}+\frac{\rho}{2} \boldsymbol{M}_{-}^{T} \boldsymbol{E}^{k+1} .
$$

Using $\boldsymbol{D}=\frac{1}{4} \boldsymbol{M}_{-} \boldsymbol{M}_{-}^{T}+\frac{1}{4} \boldsymbol{M}_{+} \boldsymbol{M}_{+}^{T}, \boldsymbol{A}=\frac{1}{4} \boldsymbol{M}_{+} \boldsymbol{M}_{+}^{T}-$ $\frac{1}{4} M_{-} M_{-}^{T}$ and (93), we can write (91) as

$$
\begin{aligned}
& \nabla f\left(\boldsymbol{\theta}^{k+1}\right)+\boldsymbol{M}_{-} \boldsymbol{\beta}^{k+1}+\rho \boldsymbol{C}\left(\boldsymbol{E}^{k}-\boldsymbol{\theta}^{k}\right) \\
& \quad+\rho\left(\boldsymbol{C}^{T}-\frac{1}{2} \boldsymbol{M}_{-} \boldsymbol{M}_{-}^{T}\right) \boldsymbol{E}^{k+1}+\rho\left(\boldsymbol{A}-\boldsymbol{C}^{T}\right) \boldsymbol{\theta}^{k+1}=\mathbf{0} .
\end{aligned}
$$

Using that $\nabla f\left(\boldsymbol{\theta}^{\star}\right)+\boldsymbol{M}_{-} \boldsymbol{\beta}^{\star}=\mathbf{0}$ and $\boldsymbol{A}=\boldsymbol{C}+\boldsymbol{C}^{T}$, we can write

$$
\begin{aligned}
\nabla f\left(\boldsymbol{\theta}^{k+1}\right)-\nabla f\left(\boldsymbol{\theta}^{\star}\right) \\
=\boldsymbol{M}_{-}\left(\boldsymbol{\beta}^{\star}-\boldsymbol{\beta}^{k+1}\right)+\rho \boldsymbol{C}\left(\boldsymbol{\theta}^{k}-\boldsymbol{\theta}^{k+1}\right)-\rho \boldsymbol{C} \boldsymbol{E}^{k} \\
\quad+\rho\left(\frac{1}{2} \boldsymbol{M}_{-} \boldsymbol{M}_{-}^{T}-\boldsymbol{C}^{T}\right) \boldsymbol{E}^{k+1},
\end{aligned}
$$

then, multiplying both sides by $\boldsymbol{\theta}^{k+1}-\boldsymbol{\theta}^{\star}$, we get

$$
\mathbb{E}\left[\left\langle\nabla f\left(\boldsymbol{\theta}^{k+1}\right)-\nabla f\left(\boldsymbol{\theta}^{\star}\right), \boldsymbol{\theta}^{k+1}-\boldsymbol{\theta}^{\star}\right\rangle\right]
$$




$$
\begin{aligned}
= & \mathbb{E}\left[\left\langle\boldsymbol{M}_{-}\left(\boldsymbol{\beta}^{\star}-\boldsymbol{\beta}^{k+1}\right), \boldsymbol{\theta}^{k+1}-\boldsymbol{\theta}^{\star}\right\rangle\right] \\
& +\rho \mathbb{E}\left[\left\langle\boldsymbol{C}\left(\boldsymbol{\theta}^{k}-\boldsymbol{\theta}^{k+1}\right), \boldsymbol{\theta}^{k+1}-\boldsymbol{\theta}^{\star}\right\rangle\right] \\
& -\rho \mathbb{E}\left[\left\langle\boldsymbol{C} \boldsymbol{E}^{k}, \boldsymbol{\theta}^{k+1}-\boldsymbol{\theta}^{\star}\right\rangle\right] \\
& -\rho \mathbb{E}\left[\left\langle\boldsymbol{C}^{T} \boldsymbol{E}^{k+1}, \boldsymbol{\theta}^{k+1}-\boldsymbol{\theta}^{\star}\right\rangle\right] \\
& +\frac{\rho}{2} \mathbb{E}\left[\left\langle\boldsymbol{M}_{-} \boldsymbol{M}_{-}^{T} \boldsymbol{E}^{k+1}, \boldsymbol{\theta}^{k+1}-\boldsymbol{\theta}^{\star}\right\rangle\right] .
\end{aligned}
$$

The first term of the right hand side can be re-written as

$$
\begin{aligned}
\mathbb{E}[ & \left.\left\langle\boldsymbol{M}_{-}\left(\boldsymbol{\beta}^{\star}-\boldsymbol{\beta}^{k+1}\right), \boldsymbol{\theta}^{k+1}-\boldsymbol{\theta}^{\star}\right\rangle\right] \\
\stackrel{(\mathrm{a})}{=} & \mathbb{E}\left[\left\langle\boldsymbol{\beta}^{\star}-\boldsymbol{\beta}^{k+1}, \boldsymbol{M}_{-}^{T} \boldsymbol{\theta}^{k+1}\right\rangle\right] \\
\stackrel{(\mathrm{b})}{=} & -\frac{2}{\rho} \mathbb{E}\left[\left\langle\boldsymbol{\beta}^{k+1}-\boldsymbol{\beta}^{\star}, \boldsymbol{\beta}^{k+1}-\boldsymbol{\beta}^{k}\right\rangle\right] \\
& -\mathbb{E}\left[\left\langle\boldsymbol{\beta}^{\star}-\boldsymbol{\beta}^{k+1}, \boldsymbol{M}_{-}^{T} \boldsymbol{E}^{k+1}\right\rangle\right],
\end{aligned}
$$

where we have used $\boldsymbol{M}_{-}^{T} \boldsymbol{\theta}^{\star}=\mathbf{0}$ in (a) and $\boldsymbol{M}_{-}^{T} \boldsymbol{\theta}^{k+1}=$ $\frac{2}{\rho}\left(\boldsymbol{\beta}^{k+1}-\boldsymbol{\beta}^{k+1}\right)-\boldsymbol{M}_{-}^{T} \boldsymbol{E}^{k+1}$ in (b).

Expanding the first term of (97), we can write

$$
\begin{gathered}
-\frac{2 \mathbb{E}\left[\left\langle\boldsymbol{\beta}^{k+1}-\boldsymbol{\beta}^{\star}, \boldsymbol{\beta}^{k+1}-\boldsymbol{\beta}^{k}\right\rangle\right]}{\rho}=\frac{1}{\rho}\left(\mathbb{E}\left[\left\|\boldsymbol{\beta}^{k}-\boldsymbol{\beta}^{\star}\right\|_{F}^{2}\right]\right. \\
\left.-\mathbb{E}\left[\left\|\boldsymbol{\beta}^{k+1}-\boldsymbol{\beta}^{\star}\right\|_{F}^{2}\right]-\mathbb{E}\left[\left\|\boldsymbol{\beta}^{k+1}-\boldsymbol{\beta}^{k}\right\|_{F}^{2}\right]\right) .
\end{gathered}
$$

Replacing the terms derived in (97) and (98) by their expressions in (96), we obtain

$$
\begin{aligned}
\mathbb{E} & {\left[\left\langle\nabla f\left(\boldsymbol{\theta}^{k+1}\right)-\nabla f\left(\boldsymbol{\theta}^{\star}\right), \boldsymbol{\theta}^{k+1}-\boldsymbol{\theta}^{\star}\right\rangle\right] } \\
= & \frac{1}{\rho} \mathbb{E}\left[\left\|\boldsymbol{\beta}^{k}-\boldsymbol{\beta}^{\star}\right\|_{F}^{2}\right]-\frac{1}{\rho} \mathbb{E}\left[\left\|\boldsymbol{\beta}^{k+1}-\boldsymbol{\beta}^{\star}\right\|_{F}^{2}\right] \\
& -\frac{1}{\rho} \mathbb{E}\left[\left\|\boldsymbol{\beta}^{k+1}-\boldsymbol{\beta}^{k}\right\|_{F}^{2}\right]+\mathbb{E}\left[\left\langle\boldsymbol{\beta}^{k+1}-\boldsymbol{\beta}^{\star}, \boldsymbol{M}_{-}^{T} \boldsymbol{E}^{k+1}\right\rangle\right] \\
& -\rho \mathbb{E}\left[\left\langle\boldsymbol{C} \boldsymbol{E}^{k}, \boldsymbol{\theta}^{k+1}-\boldsymbol{\theta}^{\star}\right\rangle\right]-\rho \mathbb{E}\left[\left\langle\boldsymbol{C}^{T} \boldsymbol{E}^{k+1}, \boldsymbol{\theta}^{k+1}-\boldsymbol{\theta}^{\star}\right\rangle\right] \\
& +\frac{\rho}{2} \mathbb{E}\left[\left\langle\boldsymbol{M}_{-} \boldsymbol{M}_{-}^{T} \boldsymbol{E}^{k+1}, \boldsymbol{\theta}^{k+1}-\boldsymbol{\theta}^{\star}\right\rangle\right] \\
& -\rho \mathbb{E}\left[\left\langle\boldsymbol{C}\left(\boldsymbol{\theta}^{k+1}-\boldsymbol{\theta}^{k}\right), \boldsymbol{\theta}^{k+1}-\boldsymbol{\theta}^{\star}\right\rangle\right] .
\end{aligned}
$$

Using the strong convexity of the function $f$, we can lower bound the left hand side of (96)

$\mathbb{E}\left[\left\langle\nabla f\left(\boldsymbol{\theta}^{k+1}\right)-\nabla f\left(\boldsymbol{\theta}^{\star}\right), \boldsymbol{\theta}^{k+1}-\boldsymbol{\theta}^{\star}\right\rangle\right] \geq \mu \mathbb{E}\left[\left\|\boldsymbol{\theta}^{k+1}-\boldsymbol{\theta}^{\star}\right\|_{F}^{2}\right]$.

Hence, we can write

$$
\begin{aligned}
\frac{1}{\rho} \mathbb{E} & {\left[\left\|\boldsymbol{\beta}^{k+1}-\boldsymbol{\beta}^{\star}\right\|_{F}^{2}+\mu\left\|\boldsymbol{\theta}^{k+1}-\boldsymbol{\theta}^{\star}\right\|_{F}^{2}\right] } \\
\leq & \frac{1}{\rho} \mathbb{E}\left[\left\|\boldsymbol{\beta}^{k}-\boldsymbol{\beta}^{\star}\right\|_{F}^{2}\right]+\rho \mathbb{E}\left[\left\langle\boldsymbol{C}\left(\boldsymbol{\theta}^{k}-\boldsymbol{\theta}^{\star}\right), \boldsymbol{\theta}^{k+1}-\boldsymbol{\theta}^{\star}\right\rangle\right] \\
& +\rho \mathbb{E}\left[\left\langle\boldsymbol{C}\left(\boldsymbol{\theta}^{\star}-\boldsymbol{\theta}^{k+1}\right), \boldsymbol{\theta}^{k+1}-\boldsymbol{\theta}^{\star}\right\rangle\right] \\
& -\frac{1}{\rho} \mathbb{E}\left[\left\|\boldsymbol{\beta}^{k+1}-\boldsymbol{\beta}^{k}\right\|_{F}^{2}\right]+\mathbb{E}\left[\left\langle\boldsymbol{\beta}^{k+1}-\boldsymbol{\beta}^{\star}, \boldsymbol{M}_{-}^{T} \boldsymbol{E}^{k+1}\right\rangle\right] \\
& -\rho \mathbb{E}\left[\left\langle\boldsymbol{C} \boldsymbol{E}^{k}, \boldsymbol{\theta}^{k+1}-\boldsymbol{\theta}^{\star}\right\rangle\right]-\rho \mathbb{E}\left[\left\langle\boldsymbol{C}^{T} \boldsymbol{E}^{k+1}, \boldsymbol{\theta}^{k+1}-\boldsymbol{\theta}^{\star}\right\rangle\right] \\
& +\frac{\rho}{2} \mathbb{E}\left[\left\langle\boldsymbol{M}_{-} \boldsymbol{M}_{-}^{T} \boldsymbol{E}^{k+1}, \boldsymbol{\theta}^{k+1}-\boldsymbol{\theta}^{\star}\right\rangle\right] .
\end{aligned}
$$

Now, using identities (32) and (33), we get the following bounds

$$
\begin{aligned}
\mathbb{E} & {\left[\left\langle\boldsymbol{C}\left(\boldsymbol{\theta}^{\star}-\boldsymbol{\theta}^{k+1}\right), \boldsymbol{\theta}^{k+1}-\boldsymbol{\theta}^{\star}\right\rangle\right] } \\
& \leq\left(\frac{\eta_{0}}{2} \sigma_{\max }^{2}(\boldsymbol{C})+\frac{1}{2 \eta_{0}}\right) \mathbb{E}\left[\left\|\boldsymbol{\theta}^{k+1}-\boldsymbol{\theta}^{\star}\right\|_{F}^{2}\right], \\
\mathbb{E} & {\left[\left\langle\boldsymbol{C}\left(\boldsymbol{\theta}^{k}-\boldsymbol{\theta}^{\star}\right), \boldsymbol{\theta}^{k+1}-\boldsymbol{\theta}^{\star}\right\rangle\right] } \\
& \leq \frac{\eta_{1} \sigma_{\max }^{2}(\boldsymbol{C})}{2} \mathbb{E}\left[\left\|\boldsymbol{\theta}^{k}-\boldsymbol{\theta}^{\star}\right\|_{F}^{2}\right]+\frac{1}{2 \eta_{1}} \mathbb{E}\left[\left\|\boldsymbol{\theta}^{k+1}-\boldsymbol{\theta}^{\star}\right\|_{F}^{2}\right], \\
\mathbb{E} & {\left[\left\langle\boldsymbol{\beta}^{k+1}-\boldsymbol{\beta}^{\star}, \boldsymbol{M}_{-}^{T} \boldsymbol{E}^{k+1}\right\rangle\right] } \\
& \leq \frac{\eta_{2}}{2} \mathbb{E}\left[\left\|\boldsymbol{\beta}^{k+1}-\boldsymbol{\beta}^{\star}\right\|_{F}^{2}\right]+\frac{\sigma_{\max }^{2}\left(\boldsymbol{M}_{-}\right)}{2 \eta_{2}} \mathbb{E}\left[\left\|\boldsymbol{E}^{k+1}\right\|_{F}^{2}\right], \\
\mathbb{E} & {\left[\left\langle\boldsymbol{C} \boldsymbol{E}^{k}, \boldsymbol{\theta}^{\star}-\boldsymbol{\theta}^{k+1}\right\rangle\right] } \\
& \leq \frac{\eta_{3}}{2} \mathbb{E}\left[\left\|\boldsymbol{\theta}^{k+1}-\boldsymbol{\theta}^{\star}\right\|_{F}^{2}\right]+\frac{\sigma_{\max }^{2}(\boldsymbol{C})}{2 \eta_{3}} \mathbb{E}\left[\left\|\boldsymbol{E}^{k}\right\|_{F}^{2}\right], \\
\mathbb{E} & {\left[\left\langle\boldsymbol{C}^{T} \boldsymbol{E}^{k+1}, \boldsymbol{\theta}^{\star}-\boldsymbol{\theta}^{k+1}\right\rangle\right] } \\
& \leq \frac{\eta_{4}}{2} \mathbb{E}\left[\left\|\boldsymbol{\theta}^{k+1}-\boldsymbol{\theta}^{\star}\right\|_{F}^{2}\right]+\frac{\sigma_{\max }^{2}(\boldsymbol{C})}{2 \eta_{4}} \mathbb{E}\left[\left\|\boldsymbol{E}^{k+1}\right\|_{F}^{2}\right], \\
\mathbb{E} & {\left[\left\langle\boldsymbol{M}_{-} \boldsymbol{M}_{-}^{T} \boldsymbol{E}^{k+1}, \boldsymbol{\theta}^{k+1}-\boldsymbol{\theta}^{\star}\right\rangle\right] } \\
& \leq \frac{\eta_{5}}{2} \mathbb{E}\left[\left\|\boldsymbol{\theta}^{k+1}-\boldsymbol{\theta}^{\star}\right\|_{F}^{2}\right]+\frac{\sigma_{\max }^{4}\left(\boldsymbol{M}_{-}\right)}{2 \eta_{5}} \mathbb{E}\left[\left\|\boldsymbol{E}^{k+1}\right\|_{F}^{2}\right],
\end{aligned}
$$

Replacing the bounds derived in (102)-(107) in (101) and introducing $\kappa>0$, we get

$$
\begin{aligned}
(1+ & \kappa) \frac{1}{\rho} \mathbb{E}\left[\left\|\boldsymbol{\beta}^{k+1}-\boldsymbol{\beta}^{\star}\right\|_{F}^{2}\right]+\mu \mathbb{E}\left[\left\|\boldsymbol{\theta}^{k+1}-\boldsymbol{\theta}^{\star}\right\|_{F}^{2}\right] \\
\leq & \frac{1}{\rho} \mathbb{E}\left[\left\|\boldsymbol{\beta}^{k}-\boldsymbol{\beta}^{\star}\right\|_{F}^{2}\right]+\frac{\rho \sigma_{\max }^{2}(\boldsymbol{C})}{2} \eta_{1} \mathbb{E}\left[\left\|\boldsymbol{\theta}^{k}-\boldsymbol{\theta}^{\star}\right\|_{F}^{2}\right] \\
& +\rho\left(\frac{1}{2 \eta_{1}}+\frac{\eta_{3}}{2}+\frac{\eta_{4}}{2}+\frac{\eta_{5}}{4}\right) \mathbb{E}\left[\left\|\boldsymbol{\theta}^{k+1}-\boldsymbol{\theta}^{\star}\right\|_{F}^{2}\right] \\
& +\left(\frac{\eta_{2}}{2}+\frac{\kappa}{\rho}\right) \mathbb{E}\left[\left\|\boldsymbol{\beta}^{k+1}-\boldsymbol{\beta}^{\star}\right\|_{F}^{2}\right] \\
& +\rho\left(\frac{\eta_{0} \sigma_{\max }^{2}(\boldsymbol{C})}{2}+\frac{1}{2 \eta_{0}}\right) \mathbb{E}\left[\left\|\boldsymbol{\theta}^{k+1}-\boldsymbol{\theta}^{\star}\right\|_{F}^{2}\right] \\
& +\frac{\rho \sigma_{\max }^{2}(\boldsymbol{C})}{2 \eta_{3}} \mathbb{E}\left[\left\|\boldsymbol{E}^{k}\right\|_{F}^{2}\right] \\
& +\left(\frac{\sigma_{\max }^{2}\left(\boldsymbol{M}_{-}\right)}{2 \eta_{2}}+\frac{\rho \sigma_{\max }^{2}(\boldsymbol{C})}{2 \eta_{4}}+\frac{\rho \sigma_{\max }^{4}\left(\boldsymbol{M}_{-}\right)}{4 \eta_{5}}\right) \\
& \mathbb{E}\left[\left\|\boldsymbol{E}^{k+1}\right\|_{F}^{2}\right] .
\end{aligned}
$$

Using that $\left\|\boldsymbol{E}^{k+1}\right\|_{F}^{2} \leq\left\|\boldsymbol{E}^{k}\right\|_{F}^{2}$, and re-arranging the terms, we can further write

$$
\begin{gathered}
\frac{1}{\rho} \mathbb{E}\left[\left\|\boldsymbol{\beta}^{k}-\boldsymbol{\beta}^{\star}\right\|_{F}^{2}\right]-\frac{1+\kappa}{\rho} \mathbb{E}\left[\left\|\boldsymbol{\beta}^{k+1}-\boldsymbol{\beta}^{\star}\right\|_{F}^{2}\right] \\
+\frac{\rho \eta_{1} \sigma_{\max }^{2}(\boldsymbol{C})}{2} \mathbb{E}\left[\left\|\boldsymbol{\theta}^{k}-\boldsymbol{\theta}^{\star}\right\|_{F}^{2}\right]
\end{gathered}
$$




$$
\begin{aligned}
& +\left(\frac{\eta_{2}}{2}+\frac{\kappa}{\rho}\right) \mathbb{E}\left[\left\|\boldsymbol{\beta}^{k+1}-\boldsymbol{\beta}^{\star}\right\|_{F}^{2}\right] \\
& -\left[\mu-\left(\frac{\eta_{0} \sigma_{\max }^{2}(\boldsymbol{C})}{2}+\frac{1}{2 \eta_{0}}+\frac{1}{2 \eta_{1}}+\frac{\eta_{3}}{2}+\frac{\eta_{4}}{2}+\frac{\eta_{5}}{4}\right) \rho\right] \\
& \times \mathbb{E}\left[\left\|\boldsymbol{\theta}^{k+1}-\boldsymbol{\theta}^{\star}\right\|_{F}^{2}\right]+\gamma \mathbb{E}\left[\left\|\boldsymbol{E}^{k}\right\|_{F}^{2}\right] \geq 0,
\end{aligned}
$$

where $\gamma=\frac{\sigma_{\max }^{2}\left(\boldsymbol{M}_{-}\right)}{2 \eta_{2}}+\frac{\rho}{2}\left(\frac{1}{\eta_{4}}+\frac{1}{\eta_{3}}\right) \sigma_{\max }^{2}(\boldsymbol{C})+$ $\frac{\rho}{4 \eta_{5}} \sigma_{\max }^{4}\left(\boldsymbol{M}_{-}\right)>0$. Fixing $\eta_{2}=\frac{2 \kappa}{\rho}$, we get

$$
\begin{aligned}
\frac{1}{\rho} \mathbb{E} & {\left[\left\|\boldsymbol{\beta}^{k}-\boldsymbol{\beta}^{\star}\right\|_{F}^{2}\right]-(1+\kappa) \frac{1}{\rho} \mathbb{E}\left[\left\|\boldsymbol{\beta}^{k+1}-\boldsymbol{\beta}^{\star}\right\|_{F}^{2}\right] } \\
+ & \frac{\rho}{2} \eta_{1} \sigma_{\max }^{2}(\boldsymbol{C}) \mathbb{E}\left[\left\|\boldsymbol{\theta}^{k}-\boldsymbol{\theta}^{\star}\right\|_{F}^{2}\right]+\frac{2 \kappa}{\rho} \mathbb{E}\left[\left\|\boldsymbol{\beta}^{k+1}-\boldsymbol{\beta}^{\star}\right\|_{F}^{2}\right] \\
- & {\left[\mu-\left(\frac{\eta_{0} \sigma_{\max }^{2}(\boldsymbol{C})}{2}+\frac{1}{2 \eta_{0}}+\frac{1}{2 \eta_{1}}+\frac{\eta_{3}}{2}+\frac{\eta_{4}}{2}+\frac{\eta_{5}}{4}\right) \rho\right] } \\
& \times \mathbb{E}\left[\left\|\boldsymbol{\theta}^{k+1}-\boldsymbol{\theta}^{\star}\right\|_{F}^{2}\right]+\gamma \mathbb{E}\left[\left\|\boldsymbol{E}^{k}\right\|_{F}^{2}\right] \geq 0 .
\end{aligned}
$$

In order to bound the term $\mathbb{E}\left[\left\|\boldsymbol{\beta}^{k+1}-\boldsymbol{\beta}^{\star}\right\|_{F}^{2}\right]$ in the left hand side, we use (95) to write

$$
\begin{aligned}
& \mathbb{E}\left[\left\|\boldsymbol{M}_{-}\left(\boldsymbol{\beta}^{\star}-\boldsymbol{\beta}^{k+1}\right)\right\|_{F}^{2}\right] \\
&= \mathbb{E}\left[\| \nabla f\left(\boldsymbol{\theta}^{k+1}\right)-\nabla f\left(\boldsymbol{\theta}^{\star}\right)+\rho \boldsymbol{C}\left(\boldsymbol{\theta}^{k+1}-\boldsymbol{\theta}^{k}\right)\right. \\
&\left.+\rho \boldsymbol{C} \boldsymbol{E}^{k}+\rho\left(\boldsymbol{C}^{T}-\frac{1}{2} \boldsymbol{M}_{-} \boldsymbol{M}_{-}^{T}\right) \boldsymbol{E}^{k+1} \|_{F}^{2}\right] .
\end{aligned}
$$

Using identity (30), we can further write

$$
\begin{aligned}
\mathbb{E} & {\left[\left\|\boldsymbol{M}_{-}\left(\boldsymbol{\beta}^{\star}-\boldsymbol{\beta}^{k+1}\right)\right\|_{F}^{2}\right] } \\
\leq & 2 \mathbb{E}\left[\left\|\nabla f\left(\boldsymbol{\theta}^{k+1}\right)-\nabla f\left(\boldsymbol{\theta}^{\star}\right)+\rho \boldsymbol{C}\left(\boldsymbol{\theta}^{k+1}-\boldsymbol{\theta}^{k}\right)\right\|_{F}^{2}\right] \\
& +2 \mathbb{E}\left[\left\|\rho \boldsymbol{C} \boldsymbol{E}^{k}+\rho\left(\boldsymbol{C}^{T}-\frac{1}{2} \boldsymbol{M}_{-} \boldsymbol{M}_{-}^{T}\right) \boldsymbol{E}^{k+1}\right\|_{F}^{2}\right] .
\end{aligned}
$$

Using (34) for the first term and (30) for the second term of the right hand side, we get

$$
\begin{aligned}
\mathbb{E} & {\left[\left\|\boldsymbol{M}_{-}\left(\boldsymbol{\beta}^{k+1}-\boldsymbol{\beta}^{\star}\right)\right\|_{F}^{2}\right] } \\
\leq & 2 \eta \mathbb{E}\left[\left\|\nabla f\left(\boldsymbol{\theta}^{k+1}\right)-\nabla f\left(\boldsymbol{\theta}^{\star}\right)\right\|_{F}^{2}\right] \\
& +\frac{2 \eta}{\eta-1} \mathbb{E}\left[\left\|\rho \boldsymbol{C}\left(\boldsymbol{\theta}^{k+1}-\boldsymbol{\theta}^{k}\right)\right\|_{F}^{2}\right]+4 \mathbb{E}\left[\left\|\rho \boldsymbol{C} \boldsymbol{E}^{k}\right\|_{F}^{2}\right] \\
& +4 \mathbb{E}\left[\left\|\rho\left(\boldsymbol{C}^{T}-\frac{1}{2} \boldsymbol{M}_{-} \boldsymbol{M}_{-}^{T}\right) \boldsymbol{E}^{k+1}\right\|_{F}^{2}\right] .
\end{aligned}
$$

Now, we can write $\mathbb{E}\left[\left\|\boldsymbol{M}_{-}\left(\boldsymbol{\beta}^{k+1}-\boldsymbol{\beta}^{\star}\right)\right\|_{F}^{2}\right] \geq$ $\tilde{\sigma}_{\min }^{2}\left(\boldsymbol{M}_{-}\right) \quad \mathbb{E}\left[\left\|\boldsymbol{\beta}^{k+1}-\boldsymbol{\beta}^{\star}\right\|_{F}^{2}\right] \quad$ where $\quad \tilde{\sigma}_{\min }\left(\boldsymbol{M}_{-}\right)$ is the minimum non-zero singular value of $M_{-}$ since both $\boldsymbol{\beta}^{k+1}$ and $\boldsymbol{\beta}^{\star}$ belong to the columns space of $M_{-}$and from Assumption 5, we have $\mathbb{E}\left[\left\|\nabla f\left(\boldsymbol{\theta}^{k+1}\right)-\nabla f\left(\boldsymbol{\theta}^{\star}\right)\right\|_{F}\right] \leq L \quad \mathbb{E}\left[\left\|\boldsymbol{\theta}^{k+1}-\boldsymbol{\theta}^{\star}\right\|_{F}\right]$. Therefore, we get

$$
\begin{aligned}
& \mathbb{E}\left[\left\|\boldsymbol{\beta}^{k+1}-\boldsymbol{\beta}^{\star}\right\|_{F}^{2}\right] \\
& \leq \frac{2 \eta}{\tilde{\sigma}_{\min }^{2}\left(\boldsymbol{M}_{-}\right)}\left(L^{2}+\frac{2 \rho^{2}}{\eta-1} \sigma_{\max }^{2}(\boldsymbol{C})\right) \mathbb{E}\left[\left\|\boldsymbol{\theta}^{k+1}-\boldsymbol{\theta}^{\star}\right\|_{F}^{2}\right]
\end{aligned}
$$

$$
\begin{aligned}
& +\frac{4 \eta \rho^{2} \sigma_{\max }^{2}(\boldsymbol{C})}{(\eta-1) \tilde{\sigma}_{\min }^{2}\left(\boldsymbol{M}_{-}\right)} \mathbb{E}\left[\left\|\boldsymbol{\theta}^{k}-\boldsymbol{\theta}^{\star}\right\|_{F}^{2}\right]+\frac{16 N \rho^{2}}{\tilde{\sigma}_{\min }^{2}\left(\boldsymbol{M}_{-}\right)} \\
& \left(\sigma_{\max }^{2}(\boldsymbol{C})+\sigma_{\max }^{2}\left(\boldsymbol{C}^{T}-\frac{1}{2} \boldsymbol{M}_{-} \boldsymbol{M}_{-}^{T}\right)\right) \psi^{2 k}
\end{aligned}
$$

where we have used that $\mathbb{E}\left[\left\|\boldsymbol{E}^{k+1}\right\|_{F}^{2}\right] \leq \mathbb{E}\left[\left\|\boldsymbol{E}^{k}\right\|_{F}^{2}\right] \leq$ $4 C_{0}^{2} N \psi^{2 k}$. Plugging the bound obtained from (114) in (110) we get

$$
\begin{aligned}
& \frac{1}{\rho} \mathbb{E}\left[\left\|\boldsymbol{\beta}^{k}-\boldsymbol{\beta}^{\star}\right\|_{F}^{2}\right]-(1+\kappa) \frac{1}{\rho} \mathbb{E}\left[\left\|\boldsymbol{\beta}^{k+1}-\boldsymbol{\beta}^{\star}\right\|_{F}^{2}\right] \\
& +\left(b_{1}+a \kappa\right) \rho \mathbb{E}\left[\left\|\boldsymbol{\theta}^{k}-\boldsymbol{\theta}^{\star}\right\|_{F}^{2}\right] \\
& \quad-\left(\mu-\frac{c \kappa}{\rho}-\left(b_{2}+a \kappa\right) \rho\right) \mathbb{E}\left[\left\|\boldsymbol{\theta}^{k+1}-\boldsymbol{\theta}^{\star}\right\|_{F}^{2}\right] \\
& +\nu \psi^{2 k} \geq 0,
\end{aligned}
$$

where $b_{1}=\frac{\eta_{1} \sigma_{\max }^{2}(\boldsymbol{C})}{2}, b_{2}=\frac{\eta_{0}}{2} \sigma_{\max }^{2}(\boldsymbol{C})+\frac{1}{2 \eta_{0}}+\frac{1}{2 \eta_{1}}+$ $\frac{\eta_{3}}{2}+\frac{\eta_{4}}{2}+\frac{\eta_{5}}{4}, c=\frac{4 \eta L^{2}}{\tilde{\sigma}_{\min }^{2}\left(\boldsymbol{M}_{-}\right)}, a=\frac{8 \eta \sigma_{\max }^{2}(\boldsymbol{C})}{(\eta-1) \tilde{\sigma}_{\min }^{2}\left(\boldsymbol{M}_{-}\right)}$, and $\nu=$ $4 N \gamma+\frac{32 N \rho \kappa}{\tilde{\sigma}_{\min }^{2}\left(\boldsymbol{M}_{-}\right)}\left(\sigma_{\max }^{2}(\boldsymbol{C})+\sigma_{\max }^{2}\left(\boldsymbol{C}^{T}-\frac{1}{2} \boldsymbol{M}_{-} \boldsymbol{M}_{-}^{T}\right)\right)$. To ensure that there is a decrease in the optimality gap, we need to determine, for which values of $\rho$, we have $c-$ $b_{2} \rho-a \rho^{2}>0$ and $\mu-\frac{c \kappa}{\rho}-\left(b_{2}+a \kappa\right) \rho \geq(1+\kappa)\left(b_{1}+a \kappa\right) \rho>0$. In other words, we need to look for $\rho$ such that

$$
-\left[\left(b_{2}+a \kappa\right)+(1+\kappa)\left(b_{1}+a \kappa\right)\right] \rho^{2}+\mu \rho-c \kappa \geq 0 .
$$

The discriminant of the quadratic equation is $\Delta=\mu^{2}-$ $4 c \kappa\left[\left(b_{2}+a \kappa\right)+(1+\kappa)\left(b_{1}+a \kappa\right)\right]$. To ensure that we can find $\rho$ such that (116) is satisfied, we need to impose that $\Delta>0$. Since $\Delta$ is a third order equation in $\kappa$, finding, for which values of $\kappa>0, \Delta>0$ is not straightforward. However, since when $\kappa \rightarrow 0, \Delta \rightarrow \mu^{2}>0$, and knowing that $\Delta$ is a decreasing function with $\Delta \rightarrow-\infty$ as $\kappa \rightarrow \infty$, then we deduce that there exits $\bar{\kappa}>0$ such that for $0<\kappa<\bar{\kappa}$, we have $\Delta>0$. In the remainder, we consider $\kappa$ such that $0<\kappa<\bar{\kappa}$. Thus, for $0<\rho<\bar{\rho}$, (116) holds where $\bar{\rho}=$ $\frac{\mu+\sqrt{\Delta}}{\left(b_{2}+a \kappa\right)+(1+\kappa)\left(b_{1}+a \kappa\right)}$

Therefore, we can write

$$
\begin{aligned}
\frac{1}{\rho} \mathbb{E} & {\left[\left\|\boldsymbol{\beta}^{k}-\boldsymbol{\beta}^{\star}\right\|_{F}^{2}\right]-(1+\kappa) \frac{1}{\rho} \mathbb{E}\left[\left\|\boldsymbol{\beta}^{k+1}-\boldsymbol{\beta}^{\star}\right\|_{F}^{2}\right] } \\
& +\rho\left(b_{1}+a \kappa\right) \mathbb{E}\left[\left\|\boldsymbol{\theta}^{k}-\boldsymbol{\theta}^{\star}\right\|_{F}^{2}\right] \\
& -\rho(1+\kappa)\left(b_{1}+a \kappa\right) \mathbb{E}\left[\left\|\boldsymbol{\theta}^{k+1}-\boldsymbol{\theta}^{\star}\right\|_{F}^{2}\right]+\nu \psi^{2 k} \geq 0 .
\end{aligned}
$$

Re-arranging the terms, we get

$$
\begin{aligned}
& \frac{1}{\rho} \mathbb{E}\left[\left\|\boldsymbol{\beta}^{k+1}-\boldsymbol{\beta}^{\star}\right\|_{F}^{2}\right]+\rho\left(b_{1}+a \kappa\right) \mathbb{E}\left[\left\|\boldsymbol{\theta}^{k+1}-\boldsymbol{\theta}^{\star}\right\|_{F}^{2}\right] \\
& \leq \frac{1}{1+\kappa}\left(\frac{1}{\rho} \mathbb{E}\left[\left\|\boldsymbol{\beta}^{k}-\boldsymbol{\beta}^{\star}\right\|_{F}^{2}\right]+\rho\left(b_{1}+a \kappa\right) \mathbb{E}\left[\left\|\boldsymbol{\theta}^{k}-\boldsymbol{\theta}^{\star}\right\|_{F}^{2}\right]\right) \\
& \quad+\frac{\nu}{1+\kappa} \psi^{2 k} .
\end{aligned}
$$


Using this equation iteratively, we can write

$$
\begin{gathered}
\frac{1}{\rho} \mathbb{E}\left[\left\|\boldsymbol{\beta}^{k+1}-\boldsymbol{\beta}^{\star}\right\|_{F}^{2}\right]+\rho\left(b_{1}+a \kappa\right) \mathbb{E}\left[\left\|\boldsymbol{\theta}^{k+1}-\boldsymbol{\theta}^{\star}\right\|_{F}^{2}\right] \\
\leq\left(\frac{1}{1+\kappa}\right)^{k+1}\left(\frac{1}{\rho} \mathbb{E}\left[\left\|\boldsymbol{\beta}^{0}-\boldsymbol{\beta}^{\star}\right\|_{F}^{2}\right]\right. \\
\left.+\rho\left(b_{1}+a \kappa\right) \mathbb{E}\left[\left\|\boldsymbol{\theta}^{0}-\boldsymbol{\theta}^{\star}\right\|_{F}^{2}\right]\right) \\
+\nu \sum_{j=0}^{k}\left(\frac{1}{1+\kappa}\right)^{k-j+1} \psi^{2 j} .
\end{gathered}
$$

Defining $\delta_{1}=\min \left\{(1+\kappa)^{-1}, \psi^{2}\right\}$ and $\delta_{2}=\max \{(1+$ $\left.\kappa)^{-1}, \psi^{2}\right\}$, we can further write

$$
\begin{aligned}
& \frac{1}{\rho} \mathbb{E}\left[\left\|\boldsymbol{\beta}^{k+1}-\boldsymbol{\beta}^{\star}\right\|_{F}^{2}\right]+\rho\left(b_{1}+a \kappa\right) \mathbb{E}\left[\left\|\boldsymbol{\theta}^{k+1}-\boldsymbol{\theta}^{\star}\right\|_{F}^{2}\right] \\
& \stackrel{(\mathrm{a})}{\leq}\left(\frac{1+\delta_{2}}{2}\right)^{k+1}\left(\frac{1}{\rho} \mathbb{E}\left[\left\|\boldsymbol{\beta}^{0}-\boldsymbol{\beta}^{\star}\right\|_{F}^{2}\right]\right. \\
& \left.+\rho\left(b_{1}+a \kappa\right) \mathbb{E}\left[\left\|\boldsymbol{\theta}^{0}-\boldsymbol{\theta}^{\star}\right\|_{F}^{2}\right]\right) \\
& +\nu \sum_{j=0}^{k}\left(\frac{1+\delta_{2}}{2}\right)^{k-j+1} \delta_{1}^{j} \\
& \stackrel{(\mathrm{b})}{\leq}\left(\frac{1+\delta_{2}}{2}\right)^{k+1}\left(\frac{1}{\rho} \mathbb{E}\left[\left\|\boldsymbol{\beta}^{0}-\boldsymbol{\beta}^{\star}\right\|_{F}^{2}\right]\right. \\
& \left.\quad+\rho\left(b_{1}+a \kappa\right) \mathbb{E}\left[\left\|\boldsymbol{\theta}^{0}-\boldsymbol{\theta}^{\star}\right\|_{F}^{2}\right]+\frac{\nu\left(1+\delta_{2}\right)}{1+\delta_{2}-2 \delta_{1}}\right),
\end{aligned}
$$

where we have used in (a) the fact that $\delta_{2} \leq\left(1+\delta_{2}\right) / 2$ since $\kappa>0$ and $\psi \in(0,1)$ and $\left(2 \delta_{1}\right) /\left(1+\delta_{2}\right) \in(0,1)$ in (b). Since $\left(1+\delta_{2}\right) / 2 \in(0,1)$, then we deduce that the sequence $\left(\boldsymbol{\theta}^{k}, \boldsymbol{\beta}^{k}\right)$ converges to $\left(\boldsymbol{\theta}^{\star}, \boldsymbol{\beta}^{\star}\right)$ linearly with a rate $\left(1+\delta_{2}\right) / 2$.

\section{REFERENCES}

[1] J. Park, S. Samarakoon, M. Bennis, and M. Debbah, "Wireless network intelligence at the edge," Proc. IEEE, vol. 107, no. 11, pp. 2204-2239, Oct. 2019.

[2] University of Oulu. $6 G$ Flagship. Accessed: Dec. 4, 2018. [Online]. Available: https://www.oulu.fi/6gflagship

[3] G. Zhu, D. Liu, Y. Du, C. You, J. Zhang, and K. Huang, "Toward an intelligent edge: Wireless communication meets machine learning," IEEE Commun. Mag., vol. 58, no. 1, pp. 19-25, Jan. 2020.

[4] J. Park et al., "Extreme URLLC: Vision, challenges, and key enablers," 2020, arXiv:2001.09683.

[5] J. Park et al., "Communication-efficient and distributed learning over wireless networks: Principles and applications," 2020, arXiv:2008.02608.

[6] M. Chen, U. Challita, W. Saad, C. Yin, and M. Debbah, "Machine learning for wireless networks with artificial intelligence: A tutorial on neural networks," IEEE Commun. Surveys Tuts., vol. 21, no. 4, pp. 3039-3071, 4th Quart., 2019.

[7] P. Kairouz et al., "Advances and open problems in federated learning," 2019, arXiv:1912.04977.

[8] H. Kim, J. Park, M. Bennis, and S.-L. Kim, "Blockchained on-device federated learning," IEEE Commun. Lett., vol. 24, no. 6, pp. 1279-1283, Jun. 2020.

[9] A. Nedić, A. Olshevsky, and M. G. Rabbat, "Network topology and communication-computation tradeoffs in decentralized optimization," Proc. IEEE, vol. 106, no. 5, pp. 953-976, Apr. 2018.

[10] K. Scaman, F. Bach, S. Bubeck, L. Massoulié, and Y. T. Lee, "Optimal algorithms for non-smooth distributed optimization in networks," in Proc. Adv. Neural Inf. Process. Syst., 2018, pp. 2740-2749.
[11] A. Elgabli, J. Park, A. S. Bedi, M. Bennis, and V. Aggarwal, "GADMM: Fast and communication efficient framework for distributed machine learning," J. Mach. Learn. Res., vol. 21, no. 76, pp. 1-39, 2020.

[12] A. Elgabli, J. Park, A. S. Bedi, C. B. Issaid, M. Bennis, and V. Aggarwal, "Q-GADMM: Quantized group ADMM for communication efficient decentralized machine learning," IEEE Trans. Commun., vol. 69, no. 1, pp. 164-181, Jan. 2021.

[13] Y. Liu, W. Xu, G. Wu, Z. Tian, and Q. Ling, "Communication-censored ADMM for decentralized consensus optimization," IEEE Trans. Signal Process., vol. 67, no. 10, pp. 2565-2579, Mar. 2019.

[14] S. Boyd, N. Parikh, E. Chu, B. Peleato, and J. Eckstein, "Distributed optimization and statistical learning via the alternating direction method of multipliers," Found. Trends Mach. Learn., vol. 3, no. 1, pp. 1-122, Jan. 2011.

[15] J. Konečný, H. B. McMahan, D. Ramage, and P. Richtárik, "Federated optimization: Distributed machine learning for on-device intelligence," 2016, arXiv:1610.02527.

[16] A. Elgabli, J. Park, C. B. Issaid, and M. Bennis, "Harnessing wireless channels for scalable and privacy-preserving federated learning," 2020, arXiv:2007.01790.

[17] H. Yu, R. Jin, and S. Yang, "On the linear speedup analysis of communication efficient momentum SGD for distributed non-convex optimization," 2019, arXiv:1905.03817.

[18] I. Gitman, H. Lang, P. Zhang, and L. Xiao, "Understanding the role of momentum in stochastic gradient methods," in Proc. Adv. Neural Inf. Process. Syst., 2019, pp. 9633-9643.

[19] H. Gao and H. Huang, "Adaptive serverless learning," 2020 , arXiv:2008.10422.

[20] K. Mishchenko, F. Hanzely, and P. Richtarik, "99\% of worker-master communication in distributed optimization is not needed," Proc. Mach. Learn. Res., vol. 124, pp. 979-988, Aug. 2020.

[21] T. Chen, G. Giannakis, T. Sun, and W. Yin, "LAG: Lazily aggregated gradient for communication-efficient distributed learning," in Proc. $A d v$. Neural Inf. Process. Syst., 2018, pp. 5050-5060.

[22] N. Singh, D. Data, J. George, and S. Diggavi, "SPARQ-SGD: Eventtriggered and compressed communication in decentralized stochastic optimization," 2019, arXiv:1910.14280.

[23] J. Bernstein, Y.-X. Wang, K. Azizzadenesheli, and A. Anandkumar, "SignSGD: Compressed optimisation for non-convex problems," in Proc. Int. Conf. Mach. Learn. (ICML), Stockholm, Sweden, vol. 80, 2018, pp. $560-569$.

[24] D. Alistarh, D. Grubic, J. Li, R. Tomioka, and M. Vojnovic, "QSGD: Communication-efficient SGD via gradient quantization and encoding," in Proc. Adv. Neural Inf. Process. Syst., 2017, pp. 1709-1720.

[25] M. M. Amiri, D. Gunduz, S. R. Kulkarni, and H. V. Poor, "Federated learning with quantized global model updates," 2020, arXiv:2006.10672.

[26] N. Sriranga, C. R. Murthy, and V. Aggarwal, "A method to improve consensus averaging using quantized ADMM," in Proc. IEEE Int. Symp. Inf. Theory (ISIT), Jul. 2019, pp. 507-511.

[27] J. Wangni, J. Wang, J. Liu, and T. Zhang, "Gradient sparsification for communication-efficient distributed optimization," in Proc. Adv. Neural Inf. Process. Syst., 2018, pp. 1299-1309.

[28] S. U. Stich, J.-B. Cordonnier, and M. Jaggi, "Sparsified SGD with memory," in Proc. Adv. Neural Inf. Process. Syst., 2018, pp. 4447-4458.

[29] E. Jeong, S. Oh, H. Kim, J. Park, M. Bennis, and S.-L. Kim, "Communication-efficient on-device machine learning: Federated distillation and augmentation under non-IID private data," in Proc. Neural Inf. Process. Syst. Workshop Mach. Learn. Phone Consum. Devices (MLPCD), Montréal, QC, Canada, 2018.

[30] J.-H. Ahn, O. Simeone, and J. Kang, "Wireless federated distillation for distributed edge learning with heterogeneous data," in Proc. IEEE Annu. Int. Symp. Pers., Indoor Mobile Radio Commun. (PIMRC), Istanbul, Turkey, Sep. 2019, pp. 1-6.

[31] J. Sun, T. Chen, G. B. Giannakis, and Z. Yang, "Communication-efficient distributed learning via lazily aggregated quantized gradients," in Proc. Adv. Neural Inf. Process. Syst., vol. 32, 2019. [Online]. Available: https://proceedings.neurips.cc/paper/2019/hash/4e87337f366f72daa424d ae11df0538c-Abstract.html

[32] A. R. Elkordy and A. S. Avestimehr, "Secure aggregation with heterogeneous quantization in federated learning," 2020, arXiv:2009.14388.

[33] W. Shi, Q. Ling, K. Yuan, G. Wu, and W. Yin, "On the linear convergence of the ADMM in decentralized consensus optimization," IEEE Trans. Signal Process., vol. 62, no. 7, pp. 1750-1761, Apr. 2014.

[34] D. Dua and C. Graff. (2017). UCI Machine Learning Repository. [Online]. Available: https://archive.ics.uci.edu/ml 


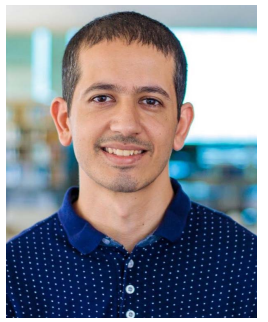

Chaouki Ben Issaid (Member, IEEE) received the Diplôme d'Ingénieur degree majors in economics and financial engineering from the Ecole Polytechnique de Tunisie (EPT) in 2013, and the master's degree in applied mathematics and computational science (AMCS) and the Ph.D. degree in statistics from the King Abdullah University of Science and Technology (KAUST) in 2015 and 2019, respectively. He is currently a Post-Doctoral Fellow with the Centre for Wireless Communications (CWC), University of Oulu. His current research interests include communication-efficient distributed learning and machine learning applications for wireless communication.

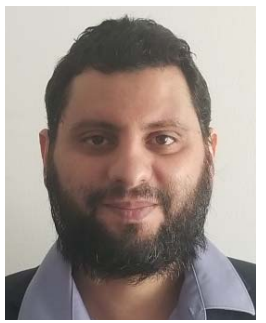

Anis Elgabli (Member, IEEE) received the B.Sc. degree in electrical and electronic engineering from the University of Tripoli, Libya, in 2004, the M.Eng. degree from UKM, Malaysia, in 2007, and the M.Sc. and Ph.D. degrees from the Department of Electrical and Computer Engineering, Purdue University, West Lafayette, IN, USA, in 2015 and 2018, respectively. $\mathrm{He}$ is currently a Post-Doctoral Researcher with the Centre for Wireless Communications, University of Oulu. His main research interests include heterogeneous networks, radio resource management, vehicular communications, video streaming, and distributed machine learning. He was a recipient of the Best Paper Award in HotSpot Workshop 2018 (Infocom 2018) and the most JUFO points in 2020 at the Center of Wireless Communication, University of Oulu.

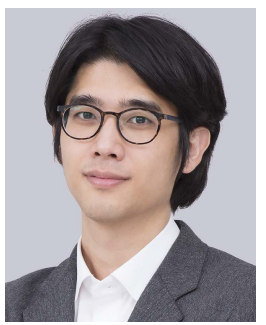

Jihong Park (Member, IEEE) received the B.S. and Ph.D. degrees from Yonsei University, Seoul, South Korea, in 2009 and 2016, respectively. He was a Post-Doctoral Researcher with Aalborg University, Denmark, from 2016 to 2017; and the University of Oulu, Finland, from 2018 to 2019. He is currently a Lecturer (an Assistant Professor) with the School of IT, Deakin University, Australia. His current research interests include communication-efficient distributed machine learning, distributed control, and distributed ledger technology, as well as their applications for beyond $5 \mathrm{G} / 6 \mathrm{G}$ communication systems. He has served as a Conference/Workshop Program Committee Member for IEEE GLOBECOM, ICC, and WCNC, as well as NeurIPS, ICML, and IJCAI. He received the IEEE GLOBECOM Student Travel Grant in 2014, the IEEE Seoul Section Student Paper Contest Bronze Prize in 2014, and the 6th IDIS-ETNEWS (The Electronic Times) Paper Contest Award sponsored by the Ministry of Science, ICT, and Future Planning of Korea. He is an Associate Editor of Frontiers in Data Science for Communications, a Review Editor of Frontiers in Aerial and Space Networks, and a Guest Editor of the Special Issue on "Millimeter Wave Communications and Networking in 5G and Beyond" of Telecom (MDPI).

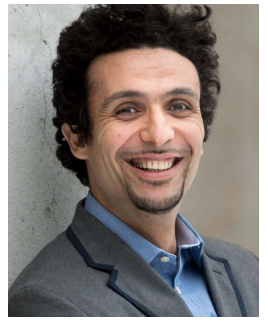

Mehdi Bennis (Fellow, IEEE) is currently a Professor with the Centre for Wireless Communications, University of Oulu, Finland, and an Academy of Finland Research Fellow and the Head of the Intelligent Connectivity and Networks/Systems Group (ICON). He has published more than 200 research papers in international conferences, journals, and book chapters. His main research interests are in radio resource management, heterogeneous networks, game theory, and distributed machine learning in $5 \mathrm{G}$ networks and beyond. He was a recipient of several prestigious awards, including the 2015 Fred W. Ellersick Prize from the IEEE Communications Society, the 2016 Best Tutorial Prize from the IEEE Communications Society, the 2017 EURASIP Best paper Award for the Journal of Wireless Communications and Networks, the all-University of Oulu Award for research, the 2019 IEEE ComSoc Radio Communications Committee Early Achievement Award, and the 2020 Clarivate Highly Cited Researcher by the Web of Science. He is an Editor of IEEE TRANSACTIONS ON COMMUNICATIONS and the Specialty Chief Editor of Data Science for Communications in the Frontiers in Communications and Networks journal.

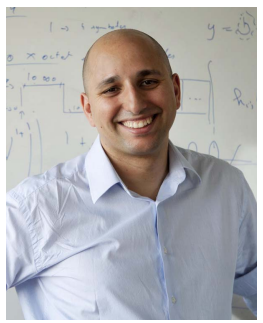

Mérouane Debbah received the M.Sc. and Ph.D. degrees from the Ecole Normale Supérieure ParisSaclay, France. He was with Motorola Labs, Saclay, France, from 1999 to 2002, and also with the Vienna Research Center for Telecommunications, Vienna, Austria, in 2003. From 2003 to 2007, he was an Assistant Professor with the Mobile Communications Department, Institut Eurecom, Sophia Antipolis, France. In 2007, he was appointed as a Full Professor with the CentraleSupelec, Gif-sur-Yvette, France. From 2007 to 2014, he was the Director of the Alcatel-Lucent Chair on Flexible Radio. From 2014 to 2021, he was the Vice President of Huawei France Research Center. He was jointly the Director of the Mathematical and Algorithmic Sciences Lab and the Lagrange Mathematical and Computing Research Center. Since 2021, he has been leading the $\mathrm{AI}$ and Telecom Systems Center at the Technology Innovation Institute. He is currently an Adjunct Professor with the Department of Machine Learning, MBZUAI. He is also the Chief Researcher with the Technology Innovation Institute, Abu Dhabi. He has managed eight EU projects and more than 24 national and international projects. His research interests lie in fundamental mathematics, algorithms, statistics, information, and communication sciences research. He is a WWRF Fellow, a Eurasip Fellow, an AAIA Fellow, an Institut Louis Bachelier Fellow, and a Membre émérite SEE. He was a recipient of the ERC Grant MORE (Advanced Mathematical Tools for Complex Network Engineering) from 2012 to 2017. He was a recipient of the Mario Boella Award in 2005, the IEEE Glavieux Prize Award in 2011, the Qualcomm Innovation Prize Award in 2012, the 2019 IEEE Radio Communications Committee Technical Recognition Award, and the 2020 SEE Blondel Medal. He received more than 20 best paper awards, among which the 2007 IEEE GLOBECOM Best Paper Award, the Wi-Opt 2009 Best Paper Award, the 2010 Newcom++ Best Paper Award, the WUN CogCom Best Paper 2012 and 2013 Award, the 2014 WCNC Best Paper Award, the 2015 ICC Best Paper Award, the 2015 IEEE Communications Society Leonard G. Abraham Prize, the 2015 IEEE Communications Society Fred W. Ellersick Prize, the 2016 IEEE Communications Society Best Tutorial Paper Award, the 2016 European Wireless Best Paper Award, the 2017 Eurasip Best Paper Award, the 2018 IEEE Marconi Prize Paper Award, the 2019 IEEE Communications Society Young Author Best Paper Award, the 2021 Eurasip Best Paper Award, the 2021 IEEE Marconi Prize Paper Award as well as Valuetools 2007, Valuetools 2008, CrownCom 2009, and Valuetools 2012, SAM 2014, and the 2017 IEEE Sweden VT-COM-IT Joint Chapter Best Student Paper Awards. $\mathrm{He}$ is an Associate Editor-in-Chief of the journal Random Matrix: Theory and Applications. He was an Associate Area Editor and a Senior Area Editor of the IEEE TRANSACTIONS ON SignAL PROCESSING from 2011 to 2013 and from 2013 to 2014, respectively. From 2021 to 2022, he serves as an IEEE Signal Processing Society Distinguished Industry Speaker. 